\title{
Trade-offs between efficiency and robustness in the empirical evaluation of asset pricing models
}

Link to publication record in Manchester Research Explorer

\section{Citation for published version (APA):}

Garrett, I., Hyde, S., \& Lozano Banda, M. (2010). Trade-offs between efficiency and robustness in the empirical evaluation of asset pricing models.

\section{Citing this paper}

Please note that where the full-text provided on Manchester Research Explorer is the Author Accepted Manuscript or Proof version this may differ from the final Published version. If citing, it is advised that you check and use the publisher's definitive version.

\section{General rights}

Copyright and moral rights for the publications made accessible in the Research Explorer are retained by the authors and/or other copyright owners and it is a condition of accessing publications that users recognise and abide by the legal requirements associated with these rights.

\section{Takedown policy}

If you believe that this document breaches copyright please refer to the University of Manchester's Takedown Procedures [http://man.ac.uk/04Y6Bo] or contact uml.scholarlycommunications@manchester.ac.uk providing relevant details, so we can investigate your claim.

\section{OPEN ACCESS}




\title{
Trade-offs between efficiency and robustness in the empirical evaluation of asset pricing models
}

\author{
Ian Garrett \\ University of Manchester* University of Manchester ${ }^{\dagger}$ \\ Martín Lozano \\ University of Manchester $\ddagger$
}

This version: 3 September 2010

\begin{abstract}
Even though we can formally express an asset pricing model in a classical Beta or in the relatively new stochastic discount factor representation, their key empirical features - notably, efficiency and robustness - may differ when estimated by the generalized method of moments. In particular, we find that there appears to be a degree of tradeoff between these features, which are linked to the asset pricing representation. Here, we investigate this issue on single and multi-factor asset pricing models using US and UK data. In this setting, our results show that the SDF approach is more likely to be less efficient but more robust than Beta method. We show that the main drivers of this trade-off are the higher order moments of the factors, in which skewness plays an important role, and especially the covariance between returns and factors.
\end{abstract}

Keywords: Empirical Asset Pricing, Factor Models, Financial Econometrics, Generalized Method of Moments, Stochastic Discount Factor, Beta pricing.

JEL Classification: C51, C52, G12.

\footnotetext{
${ }^{*}$ E-mail: ian.garrett@mbs.ac.uk

${ }^{\dagger}$ E-mail: stuart.hyde@mbs.ac.uk

${ }^{\ddagger}$ Corresponding author. E-mail: Martin.LozanoBanda@mbs.ac.uk An earlier version was presented (or accepted for presentation) at the 2008 Annual Doctoral Conference at Manchester Business School; Internal Seminar (University Carlos III de Madrid, April 2008); Jornada de Investigación en Finanzas (Universidad de Castilla-La Mancha, October 2008); XVI AEFIN Finance Forum (ESADE Business School, Barcelona, November 2008); Southwestern Finance Association 48th Annual Meeting; (Oklahoma City, February 2009); and the Eastern Finance Association Annual Meeting (Washington D.C., May 2009). We would like to thank Genaro Sucarrat (Universidad Carlos III de Madrid), Chien-Ting Lin (University of Adelaide), and Sheng Guo (Florida International University) for helpful comments as discussants, and to Raymond Kan for kindly sharing complementary econometric notes on Kan and Zhou [35].
} 


\section{Introduction}

Any asset pricing model can be formally characterized either under a Beta or a stochastic discount factor $(\mathrm{SDF})$ representation. The SDF representation, states that the value of a financial asset equals the expected value of the product of the payoff on the asset and the SDF. Whereas accordingly to the Beta representation, the expected return on an asset, is a linear function of its factor betas. This traditional approach is widely implemented in the finance literature using the two-stage cross-sectional regression methodology advocated by Black, Jensen and Scholes [5], and Fama and MacBeth [17], see for example Kan, Robotti and Shanken [32]. Yet, the relatively new SDF characterization can be traced back to Dybvig and Ingersoll [14], Hansen and Richard [23], and Ingersoll [24], who derive it for a number of theoretical asset pricing models formerly expressed under the classical Beta framework.

Even though both characterizations are theoretically equivalent, the parameters of interest are in general dissimilar under the two setups. In particular, the Beta representation is formulated to analyze the factor risk premium $\delta$, and the Jensen alpha $\alpha$. In contrast, the SDF representation is intended to analyze the parameters that enter into the imposed stochastic discount factor $\lambda$, and the pricing errors $\pi$. See Ferson and Jagannathan [20] for a general approach to this equivalence. As a matter of fact, only when the factor is standardized to have zero mean and a variance equal to one, the parameters of interests numerically coincides in both methods such as $\delta=\lambda$, and $\alpha=\pi$; however this is rather a theoretical implication than an real possibility in practice.

The fact that both representations are equivalent, imply that there is a one-to-one mapping between $\delta$ and $\lambda$; and between $\alpha$ and $\pi$ which may facilitates the comparison of the estimators. Though, this theoretical equivalence does not necessarily entail an empirically equivalence. Therefore, the experimental questions that arises are: (1) Is it better to make inferences on $\delta$ or on $\lambda$ ? And analogously: (2) Is it better to make inferences on $\alpha$ or on $\pi$ ? From now on, we will refer to the Beta and SDF methods to the generalized method 
of moments (GMM) of Hansen [21], estimation and evaluation of an asset pricing model expressed in a Beta or SDF representation.

The comparison of these methods is far from being trivial for several reasons; one of them is that the associated magnitudes of their respective parameters are not alike in general. For example, a $\widehat{\delta}$ equal to 0.6 correspond to an approximately value of 2 for $\widehat{\lambda}$ when estimating a single-factor model using US data. If we directly compare the simulated standard errors $\sigma(\widehat{\delta})$ and $\sigma(\widehat{\lambda})$, our results would be misleading simply by a scaling issue. In this paper, we take advantage of the one-to-one mapping between Beta and SDF estimators in order to transform the Beta estimators into SDF units. By doing so, we are able to perform a fair comparison of the simulated standard errors because even though the values do not numerically coincide, they will have the same measure units.

It is a common trend in empirical finance to compare the performance of different econometric procedures within the Beta framework, or within the SDF method. For example, Jagannathan and Wang [28] compare the asymptotic efficiency of the two-stage cross-sectional regression and the Fama-MacBeth procedure. Shanken and Zhou [44], analyze the finite sample properties and empirical performance of the Fama-MacBeth, maximum likelihood, and GMM for Beta pricing models. Other related examples can be found in Farnsworth, Ferson, Jackson and Todd [18], Velu and Zhou [48], Kan and Robotti ([31], [30]), Chen and Kan [11], and Amsler and Schmidt [2], just to mention a few. However, only recently have there been attempts to evaluate the performance in finite samples of the Beta versus the SDF approaches, and this is where our main interest rely because it has become a rich research field in financial econometrics and asset pricing.

The curiosity surrounding this topic responds to several factors. The asset pricing models are widely used in empirical analysis not only because of the abundance of available financial statistical data, but because they can be applied extensively in practical research and decision making processes. For instance, researchers and practitioners may employ them for calculations of costs of capital associated with investment and takeover decisions, which it is a recurrent task in areas such as accountability and corporate finance. Also, 
asset pricing models are used in comparative analysis of the success of different investors or performance evaluation of investment funds. They are even applied in judicial inquires related to court decisions regarding compensation to expropriated firms whose shares are not listed on the stock market. Then, the performance of the Beta and SDF methods becomes relevant because as far as the chosen technique delivers more precise estimators, the calculations and hypothesis tests results will be more trustworthy. For example, in order to evaluate an investment project, managers have to discount the project's future cash flows. To do so, managers require a projection of the cash flows and an estimation of a discount rate. It follows that if this estimation is not accurate enough, the evaluation of the investment project will not be assertive either. For other related implications see Kan and Zhou ([35],[33]), Jagannathan and Wang [28], and Lozano and Rubio [39].

In the first attempt to evaluate the performance in finite samples of the Beta versus the SDF approaches, and using a standardized single-factor model, Kan and Zhou [35] show that the SDF method is much less efficient than the Beta method. Jagannathan and Wang [28], and Cochrane $([12],[13])$, debate this conclusion in a non-standardized single-factor model and based on joint normality assumption for both the asset returns and the factors. They conclude that the SDF method is as efficient as the Beta method for estimating risk premiums. In addition, they find that the specification tests are equally powerful. Short after, in a non-published manuscript, Kan and Zhou [33] show that, under a more general distributional assumption, and considering non-standardized factors, inference based on $\lambda$ can still be less reliable than inference based on $\delta$ in realistic situations where the factors are leptokurtic. Contributing to the discussion, Ferson [19] concludes that when the two methods correctly exploit the same moments they deliver nearly identical results. The interest about the topic has recently risen and attracted other researchers. For example, Lozano and Rubio [39] show evidence suggesting that inference on $\delta$ and $\pi$, is more reliable than inference on their correspondent estimators $\lambda$ and $\alpha$. On the other hand, Sentana and Peñaranda [42] show that a particular GMM procedure gives rise to numerically identical Beta and SDF estimates. 
In this paper, we advocate that the core difference between the SDF and the Beta methods is a matter of trade-off between efficiency versus robustness. Commonly in econometrics, a robustness approach places fewer restrictions to the estimator. In this sense, the SDF method does not place any restriction to $\lambda$, whereas the Beta method explicitly incorporates the definition of $\delta$ as a subset of the moment restrictions. Therefore, it may be reasonable to expect that Beta method should be more efficient than SDF method and, conversely, SDF method should be more robust than Beta method.

Regarding the Beta method, there are no disagreement about which set of moments have to be included in the GMM estimation. However, there are two dominant and subtly different SDF representations in the literature. The first one defines the SDF as a linear function of the factors. Nonetheless, Kan and Robotti [31] point out that this is problematic because the specification test statistic is not invariant to an affine transformation of the factors. Therefore, in our study we also consider an alternative specification that defines it as a linear function of de-meaned factors. These two SDF variants are also known as uncentred and centred respectively. The methods are then applied to estimate and evaluate the single-factor CAPM of Sharpe [45], Lintner [38] and Mossin [41]; the three factor FamaFrench [16]; the four factor Carhart [10], and the three factor RUH of Lozano and Rubio [39] models in an application to US data. We also provide some illustrative results using a reduced UK sample.

There are two more differences in our finite sample approach with respect to previous similar studies that raise a larger number of implications and original results. The first is that we assume factors and returns are drawn from their empirical distribution. The second is that we evaluate not only single, but multi-factor linear asset pricing models. By doing so, we allow the methods to perform under the presence of notably non-normal distributions, as it actually happen in more realistic situations. Furthermore, we use a diverse number of test assets in order to address the tight factor structure problem advocated by Lewellen, Nagel and Shanken [36].

The debate about the differences regarding both methodologies is still far from being 
conclusive. Furthermore, the available results in the current literature normally rely on the evaluation of the single-factor model which is still an important benchmark. In spite of this, multi-factor pricing models are by far the most commonly used in current empirical applications, and they incorporate factors with greater non-normalities than the market factor itself. For instance, in our sample, momentum factor has similar mean and variance as the market factor; nonetheless it is almost three times more leptokurtic. Likewise, market factor is close to be symmetric, although skewness for size and value factors is positive, but markedly negative for momentum. See Table 10 for the full descriptive statistics for factors and portfolios used in this paper.

Highly influential works in this field fail to evaluate the methods under harder conditions such as the high non-normalities present in others extensively used factors such as size, value and momentum. Presumably, the conclusions of evaluating the methods under the single-factor model - and imposing distributional assumptions - would not be necessarily valid when the evaluation considers multi-factor models which data is generated from their empirical distributions. Therefore our results regarding multi-factor models would contribute to cover this gap in the current literature, and they may be comparable to a wider set of applications in finance as well. There are other kinds of models such as the non-linear, often used for pricing derivative securities like futures and options; however, they can be frequently linearized and treated as multi-factor models as well. Consequently, our results regarding multi-factor models imply a considerably wider range of applicability compared to previous studies.

We show that, in general, the Beta method is more efficient but less robust than the SDF method. That is, $\widehat{\delta}$ and $\widehat{\pi}$ have lower simulated standard errors than their correspondent $\widehat{\lambda}$ and $\widehat{\alpha}$. We illustrate that the choice of the Beta or the SDF method can be condensed into a trade-off between efficiency versus robustness. In this sense, we provide empirically motivated evidence about what drives this trade-off. This easy to validate information is valuable for researchers and practitioners because they would have an a priori rough idea about the relative benefits or pitfalls of following either method in order to conduct 
empirical analysis.

We show that the factor negative skewness - which is usually presented in momentum and the low covariances or second moment matrix of returns and factors, potentially constitutes a drawback to the SDF method at estimating $\lambda$ even if the sample is as large as one thousand. Additionally, the application to the UK data illustrate that this relative disadvantage increases as we consider small samples. In any case, this drawback at estimating $\lambda$ represent an advantage at estimating $\pi$ given that the SDF method is basically devoted to minimize the pricing errors.

We conclude that the choice of estimate and evaluate an asset pricing model by either method, can be reduced to a choice between efficiency versus robustness. Therefore, we argue that the selection should depend on the experiential purpose, or more specifically, on the parameter of interest. In particular, if the main interest relies on the inference of the estimator, the Beta method would likely provide more accurate estimates. On the other hand, if the main concern is to achieve lower pricing errors, the SDF method should be implemented. Hence, there is no method that fully dominates the other, rather they are complementary, and they should not be considered as empirically equivalent.

The outline of the remainder of the paper is as follows. In section 2 we present the methodology, section 3 presents the results, while section 4 concludes. Finally, the Tables and Figures are in 5 and appendix is in section 6.

\section{Methodology}

In order to estimate and evaluate the Beta and SDF representations we follow the GMM econometric procedure of Hansen [21]. Therefore we would retrieve valid inferences even if the assumptions of serial independence, conditional homoskedasticity or normality are not wholly realistic in practice. For a deeper discussion about the GMM applied to the estimation and evaluation of asset pricing models see Campell, Lo and MacKinlay [9], Jagannathan, Skoulakis and Wang [25], and Cochrane [13]. 


\subsection{The Beta method}

We denote $r_{t}$ as the vector of $N$ stock returns in excess of the risk-free rate and $f_{t}$ a vector of $K$ economy-wide pervasive risk factors during period $t$. The mean and the covariance matrix of the factors are denoted by $\mu$, where $\mu=\mathrm{E}\left[f_{t}\right]$, and $\Omega$ respectively. The standard linear asset-pricing model under the Beta representation is given by

$$
\mathrm{E}\left[r_{t}\right]=\delta \beta
$$

where $\delta$ is the vector of factor risk premiums, and $\beta$ is the matrix of factor loadings which measure the sensitivity of asset returns to the factors, defined as

$$
\beta_{N \times K} \equiv \mathrm{E}\left[r_{t}\left(f_{t}-\mu\right)^{\prime}\right] \Omega^{-1}
$$

Equivalently, we can identify $\beta$ as a parameter in the time-series regression

$$
r_{t}=\phi+\beta f_{t}+\epsilon_{t}
$$

where the residual $\epsilon_{t}$ has zero mean and is uncorrelated with the factors $f_{t}$. The specification of the asset-pricing model under the Beta representation in equation (1) imposes the following restriction on the time-series intercept, $\phi=(\delta-\mu) \beta$. By substituting this restriction in the regression equation, we obtain:

$$
r_{t}=\left(\delta-\mu+f_{t}\right) \beta+\epsilon_{t} \quad \text { where }\left\{\begin{array}{l}
\mathrm{E}\left[\epsilon_{t}\right]=0_{N} \\
\mathrm{E}\left[\epsilon_{t} f_{t}^{\prime}\right]=0_{N \times K}
\end{array}\right.
$$

Hence, the Beta representation in equation (1) gives rise to the factor model in equation (4). The associated set of moment conditions $g$ of the factor model are:

$$
\begin{aligned}
& \mathrm{E}\left[r_{t}-\left(\delta-\mu+f_{t}\right) \beta\right]=0_{N} \\
& \mathrm{E}\left[\left[r_{t}-\left(\delta-\mu+f_{t}\right) \beta\right] f_{t}^{\prime}\right]=0_{N \times K}
\end{aligned}
$$

However, when the factor is the return on a portfolio of traded assets, as in the single and multi-factor models analyzed in this paper - the CAPM, Fama-French, RUH, and the 
Carhart factor models - it can be verified that the estimate of $\mu$ (the sample mean of the factor) is also the estimate of the risk premium $\delta$. Therefore, given $\delta=\mu$, the moment conditions given in equation (5) simplify to

$$
\begin{aligned}
& \mathrm{E}\left[r_{t}-f_{t} \beta\right]=0_{N} \\
& \mathrm{E}\left[\left(r_{t}-f_{t} \beta\right) f_{t}^{\prime}\right]=0_{N \times K} \\
& \mathrm{E}\left[f_{t}-\mu\right]=0_{K}
\end{aligned}
$$

where neither $\delta$ or $\mu$ appear in the first two restrictions of equation (6) but it is necessary to include the definition of $\mu$ to identify the vector of risk premiums $\delta$ as a third moment restriction. Nevertheless, it is also possible to estimate the last moment restriction of equation (6) outside the GMM framework by computing $\mu=E\left[f_{t}\right]$. This is because the number of added moment restrictions in equation (6) compared with equation (5) is the same as the number of added unknown parameters. Hence, the efficiency of equation (5) and equation (6) remains the same. By following this alternative, we drop the factor-mean moment condition without ignoring that it has to be estimated. An additional moment condition to estimate the variance $\Omega$ could also be added to equation (6). However the variance can also be estimated outside the GMM framework without affecting efficiency.

Now, following the usual GMM notation, we define the vector of unknown parameters $\theta=\left[\operatorname{vec}(\beta)^{\prime} \mu^{\prime}\right]^{\prime}$, where the vec operator 'vectorizes' the $\beta_{N \times K}$ matrix by stacking its columns, and the observable variables are $x_{t}=\left[\begin{array}{ll}r_{t}^{\prime} & f_{t}^{\prime}\end{array}\right]^{\prime}$. Then, the function $g$ in the moment restriction is given by

$$
g\left(x_{t}, \theta\right)_{(N+N K+K) \times 1}=\left(\begin{array}{c}
r_{t}-f_{t} \beta \\
\operatorname{vec}\left[\left(r_{t}-f_{t} \beta\right) f_{t}^{\prime}\right] \\
f_{t}-\mu
\end{array}\right)
$$

In which, for any $\theta$, the sample analogue of $\mathrm{E}\left[g\left(x_{t}, \theta\right)\right]$ is equal to

$$
g_{T}(\theta)=\frac{1}{T} \sum_{t=1}^{T} g\left(x_{t}, \theta\right)
$$

Then, a natural estimation strategy for $\theta$ is to choose the values that make $g_{T}(\theta)$ as 
close to the zero vector as possible. For that reason we choose $\theta$ to solve

$$
\min _{\theta} g_{T}(\theta)^{\prime} W^{-1} g_{T}(\theta)
$$

To compute the first-stage GMM estimator $\theta_{1}$ we consider $W=I$ in equation (9). The second-stage GMM estimator $\theta_{2}$ is the solution of equation (9) when the weighting matrix is the spectral density matrix of $g\left(x_{t}, \theta_{1}\right)$ :

$$
S=\sum_{j=-\infty}^{\infty} \mathrm{E}\left[g\left(x_{t}, \theta_{1}\right) g\left(x_{t}, \theta_{1}\right)^{\prime}\right]
$$

In order to examine the validity of the pricing model derived from the moment restrictions in equation (6) we can test whether the vector of $N$ Jensen's alphas, given by $\alpha=\mathrm{E}\left[r_{t}\right]-\delta \beta$ is jointly equal to zero. ${ }^{1}$ This can be done using the $J$-statistic with an asymptotic $\chi^{2}$ distribution. Given that there are $N+N K+K$ equations and $N K+K$ unknown parameters in equation (7), then the degrees of freedom is equal to $N$.

The covariance matrix of the pricing errors, $\operatorname{Cov}\left(g_{T}\right)$, is given by

$$
\operatorname{Cov}\left(g_{T}\right)=\frac{1}{T}\left[\left(I-\beta\left(\beta^{\prime} \beta\right)^{-1} \beta^{\prime}\right) S\left(I-\beta\left(\beta^{\prime} \beta\right)^{-1} \beta^{\prime}\right)\right]
$$

and the test is a quadratic form of the vector of pricing errors. In particular, the Hansen [21] $J$-statistic is computed as

$$
\begin{array}{ll}
\text { First-stage: } & g_{T}\left(\theta_{1}\right)^{\prime} \operatorname{Cov}\left(g_{T}\right)^{-1} g_{T}\left(\theta_{1}\right) \sim \chi_{N}^{2} \\
\text { Second-stage: } & T g_{T}\left(\theta_{2}\right)^{\prime} S^{-1} g_{T}\left(\theta_{2}\right) \sim \chi_{N}^{2}
\end{array}
$$

Both the first and second-stage statistic in equation (12) lead to the same numerical value. However, if we weight equations (11) and (12) by any other matrix different to $S$, such as $\mathrm{E}\left[r_{t} r_{t}^{\prime}\right]$ or $\operatorname{Cov}\left[r_{t}\right]$, this result no longer holds.

\footnotetext{
${ }^{1}$ This approach is known as the restricted test, see MacKinlay and Richardson [40].
} 


\subsection{The SDF method}

To derive the SDF representation from the Beta representation we follow Ferson and Jagannathan [20], and Jagannathan and Wang [28] among others. First, we substitute the expression for $\beta$ in equation (2) into equation (1) and rearrange the terms, to give:

$$
\mathrm{E}\left[r_{t}\right]-\mathrm{E}\left[r_{t} \delta^{\prime} \Omega^{-1} f_{t}-r_{t} \delta^{\prime} \Omega^{-1} \mu^{\prime}\right]=\mathrm{E}\left[r_{t}\left(1+\delta^{\prime} \Omega^{-1} \mu-\delta^{\prime} \Omega^{-1} f_{t}\right)\right]=0_{N}
$$

again, if we are considering traded factors, then $\delta=\mu$ so $1+\delta^{\prime} \Omega^{-1} \mu=1+\mu^{\prime} \Omega^{-1} \mu \geq 1$, then divide each side by $1+\delta^{\prime} \Omega^{-1} \mu,{ }^{2}$

$$
\mathrm{E}\left[r_{t}\left(1-\frac{\delta^{\prime} \Omega^{-1}}{1+\delta^{\prime} \Omega^{-1} \mu} f_{t}\right)\right]=0_{N}
$$

If we transform the vector of risk premiums $\delta$ into a vector of new parameters $\lambda$ as follows,

$$
\lambda=\frac{\delta^{\prime} \Omega^{-1}}{1+\delta^{\prime} \Omega^{-1} \mu}
$$

then we obtain the following SDF representation, which is at the same time the set of moment restrictions $g$ of the linear asset-pricing model,

$$
\mathrm{E}\left[r_{t}\left(1-\lambda f_{t}\right)\right]=0_{N}
$$

where the random variable $m_{t} \equiv 1-f_{t}^{\prime} \lambda$ is the SDF because $\mathrm{E}\left[r_{t} m_{t}\right]=0_{N}$. Alternatively, we could derive the Beta representation from the SDF representation by expanding $m$ and rearranging the terms.

From the moment restrictions, equation (14), we obtain the vector of $N$ pricing errors defined as $\pi=\mathrm{E}\left[r_{t}\right]-\lambda \mathrm{E}\left[r_{t} f_{t}\right]$. The analytical solution of equation (14) is obtained by GMM. ${ }^{3}$ Writing the sample pricing errors as

$$
g_{T}(\lambda)=-\mathrm{E}\left[r_{t}\right]+\lambda \mathrm{E}\left[r_{t} f_{t}\right]
$$

\footnotetext{
${ }^{2}$ Even when the factors are not traded, it is common to suppose $1+\delta^{\prime} \Omega^{-1} \mu \neq 0$.

${ }^{3}$ This is useful given the need to undertake vast numbers of simulations. Similar simplifications of multidimensional optimization problems for Beta models can be found in Shanken and Zhou [44].
} 
where $d=-\frac{\partial g_{T}(\lambda)}{\partial \lambda^{\prime}}=\mathrm{E}\left[r_{t} f_{t}\right]$, the second-moment matrix of returns and factors. The firstorder condition to minimize the quadratic form of the sample pricing errors, equation (9), is $-d^{\prime} W\left[\mathrm{E}\left[r_{t}\right]-\lambda d\right]=0$, where $W$ is the GMM weighting matrix, equal to the identity matrix in the first-stage estimator and equal to the spectral density matrix $S$, equation (10), in the second-stage estimator. Therefore, the GMM estimates of $\lambda$ are:

$$
\begin{aligned}
& \widehat{\lambda}_{1}^{A}=\left(d^{\prime} d\right)^{-1} d^{\prime} \mathrm{E}\left[r_{t}\right] \\
& \widehat{\lambda}_{2}^{A}=\left(d^{\prime} S^{-1} d\right)^{-1} d^{\prime} S^{-1} \mathrm{E}\left[r_{t}\right]
\end{aligned}
$$

For illustrative purposes, we decorate with an $A$ to $\hat{\lambda}$ to indicate that the estimator comes from the un-centred specification and with a $B$ to indicate that comes from the centred specification.

Specifying the SDF as a linear function of the factors as in equation (14) has been very popular in the empirical literature. However, Kan and Robotti [31] point out that this is problematic because the specification test statistic is not invariant to an affine transformation of the factors, and Burnside [7] also reach to similar conclusions. Therefore, we also consider an alternative specification that defines the SDF as a linear function of de-meaned factors. Examples of this representation can be found in Julliard and Parker [29] and Yogo $[50]$.

The alternative centred version of equation (14) is defined as:

$$
\mathrm{E}\left[r_{t}\left[1-\lambda\left(f_{t}-\mu\right)\right]\right]=0_{N}
$$

According to [26] and [28], it is also possible to estimate $\mu$ in equation (17) outside of the GMM estimation by computing $\mu=\mathrm{E}\left[f_{t}\right]$. This is because the number of added moment restrictions is the same as the number of added unknown parameters. Hence, the efficiency of the estimators remains the same. By following this alternative, we can drop the factor-mean moment condition without ignoring that it has to be estimated, and obtain analytical expressions for $\widehat{\lambda}_{1}^{B}$ and $\widehat{\lambda}_{2}^{B}$.

Naturally, the procedure to solve the moment restrictions in equation (17) is similar to that for the un-meaned $\mathrm{SDF}^{A}$ method. In particular, we substitute $\mathrm{E}\left[r_{t} f_{t}\right]$ for $\operatorname{Cov}\left[r_{t} f_{t}\right]$ 
in equation (15), then define $b=-\frac{\partial g_{T}(\lambda)}{\partial \lambda^{\prime}}$ as the covariance matrix of returns and factors. Finally, the $\mathrm{SDF}^{B}$ first and second stage GMM estimates are:

$$
\begin{aligned}
& \widehat{\lambda}_{1}^{B}=\left(b^{\prime} b\right)^{-1} b^{\prime} \mathrm{E}\left[r_{t}\right] \\
& \widehat{\lambda}_{2}^{B}=\left(b^{\prime} S^{-1} b\right)^{-1} b^{\prime} S^{-1} \mathrm{E}\left[r_{t}\right]
\end{aligned}
$$

The specification tests can be conducted by following equations (8) and (12), the only difference being that we substitute $\beta$ by $d=\mathrm{E}\left[r_{t} f_{t}\right]$ (the second moment matrix of returns and factors) for the $\mathrm{SDF}^{A}$ case, and by $b=\operatorname{Cov}\left[r_{t} f_{t}\right]$ (the covariance matrix of returns and factors) for the $\mathrm{SDF}^{B}$ case. The degrees of freedom in equation (12) are specific for the Beta method, in the SDF method the degrees of freedom is equal to $N-K$, since there are $N$ equations and $K$ unknown parameters in both equations (14) and (17).

The covariance matrix of the pricing errors and the $J$-statistic represented in equations (11) and (12) are weighted by equation (10), since it is statistically optimal. This approach was first suggested by Hansen [21] as it maximizes the asymptotic statistical information in the sample about a model, given the choice of moments. However, there are also alternatives for this weighting matrix which are suitable for model comparisons because they are invariant to the model and their parameters. For instance, Hansen and Jagannathan [22] suggest the use of the second moment matrix of excess returns $W=\mathrm{E}\left[r_{t} r_{t}^{\prime}\right]$ instead of $W=S$. Also, Burnside [7], Balduzzi and Yao [3], and Kan and Robotti [31] suggest that the $\mathrm{SDF}^{B}$ method should use the covariance matrix of excess returns $W=\operatorname{Cov}\left[r_{t}\right]$. We investigate the implications of using these alternative weighting matrices.

\subsection{Comparison of the methods}

There is a one-to-one mapping between the factor risk premium $\delta$ and the SDF parameter $\lambda$, which facilitates the comparison of the two methods. ${ }^{4}$ Hence, we can derive an estimate of $\lambda$ not only by the SDF method but also by the Beta method. By the same token we can derive an estimate of $\delta$ not only by the Beta method but also by the SDF method. From

\footnotetext{
${ }^{4}$ Thanks to Raymond Kan for kindly sharing complementary econometric notes on Kan and Zhou [33].
} 
the previous definition of $\lambda$ in equation (13), we have:

$$
\lambda=\frac{\delta}{\Omega+\delta \mu^{\prime}} \quad \text { or } \quad \delta=\frac{\Omega \lambda}{1-\mu^{\prime} \lambda}
$$

In a similar way, by substituting equation (19) into $\pi$, we can find a one-to-one mapping between $\pi$ from the SDF method and $\alpha$ from the Beta method.

$$
\pi=\frac{\Omega}{\Omega+\delta \mu^{\prime}} \alpha \quad \text { or } \quad \alpha=\frac{\Omega+\delta \mu^{\prime}}{\Omega} \pi
$$

We should not directly compare $\lambda$ versus $\delta$, neither $\pi$ versus $\alpha$ since they are measured with different units. Any direct comparison would be biased simply because a scaling issue, and not necessarily because an intrinsic differences among the Beta and SDF methods. An alternative for avoiding this scaling issue is to transform $\delta$ into $\lambda$ units, and $\alpha$ into $\pi$ units following equations (19) and (20). For convenience, we will decorate all Beta estimators with ' $*$ ' in order to identify that they are Beta estimators.

In the first formal attempt to compare both methods, Kan and Zhou [35] assume that the factor has zero mean and unit variance, that is $\mu=0$ and $\Omega=1$. In this standardized single factor model, equations (19) and (20) simplify to $\lambda=\delta$ and $\pi=\alpha$. By assuming that the mean and the variance of the factor are predetermined without estimation, they ignore the sampling errors associated with the estimates of $\mu$ and $\Omega$ and conclude that the estimates of the Beta method are more efficient. Jagannathan and Wang [28] and Cochrane [12] explain the effects of standardized factors, showing that in general, predetermining the factor moments reduces the sampling error of the estimate in the Beta method and not in the SDF method.

However, with the Beta moment restrictions, equation (6), we only can make inference on $\delta$, not on $\lambda$. Yet to compare the methods using equations (19) and (20) requires an estimator of $\Omega$. One solution is to add an additional moment condition to equation (6) to estimate $\Omega$. An alternative is to estimate $\mu$ and $\Omega$ outside the GMM estimation. In simulation results not showed here, we find that the efficiency of both alternatives is the same. Hence we elect to estimate $\Omega$ outside the GMM estimation. 
Predetermining the values of $\mu$ and $\Omega$ to be known constants - not necessarily $\mu=0$ and $\Omega=1$ - gives an informational advantage to the Beta method in terms of efficiency. Predetermining without estimation implies ignoring the sampling errors associated with $\mu^{*}$ and $\Omega^{*}$, as a consequence $\lambda^{*}$ becomes considerably more efficient than if we follow equation (6). In our simulation analysis, we consider the case where $\mu$ and $\Omega$ must be estimated.

To summarize, the Beta method gives the GMM estimate $\delta^{*}$ while the SDF method gives the GMM estimate $\lambda$. In our simulation approach, we transform the estimate $\delta^{*}$ into an estimate of $\lambda$ and then compare the variances of the sampling distribution of $\widehat{\lambda}^{*}$ and $\widehat{\lambda}$. In the same way, we transform $\alpha^{*}$ into an estimate of $\pi$ and then compare the efficiency of $\widehat{\pi}^{*}$ and $\widehat{\pi}$. We also compare the distributions of Hansen's [21] test of overidentification using the $J$-statistic of the transformed Beta $\widehat{J}^{*}$ and $\widehat{J}$ from the SDF method. The null hypothesis is that all pricing errors are zero.

\subsection{Simulation setup}

Researchers who estimate these kind of asset pricing models are frequently faced with data sets of finite, and occasionally rather small, sample sizes, see Ahn and Gadarowski [1] for an examination of finite-sample properties of several model tests methods. In the case of US, the data set can be as large as 1,000 monthly historical observations, but in the case of UK, we can end up with about 300. It is therefore imperative to obtain a sense of the small sample performance of the two methods. Since finite-sample analytical results can be obtained only under certain distributional assumptions of the returns, factors and errors, it is customary to resort to some simulation technique, which allows to alter the simulation input and develop an understanding of how sensitive the results are with respect to the various features of the data generating process.

We use Bootstrap simulation ${ }^{5}$ to find out whether the GMM estimators and test statis-

\footnotetext{
${ }^{5}$ Simulations were executed using the North West Grid (NW-Grid) computational facilities provided by The University of Manchester.
} 
tics have any bias. In particular, we are interested in evaluating the standard deviation of $\widehat{\lambda}^{*}, \widehat{\lambda}, \widehat{\pi}^{*}, \widehat{\pi}$, denoted as $\sigma(\cdot)$ and also the tail of the $\widehat{J}$-statistic distribution to conduct specification tests. We assume that the factors $f_{t}$ are drawn from their empirical distribution which allows for non-normalities, autocorrelation, heteroskedasticity and non-independence of factors and residuals.

To artificially generate the excess returns we use the factor model, equation (4) where $t=1, \ldots, T$. For $T$, we consider the following four time horizons: 60, 360, 600 and 1000 months. As Shanken and Zhou [44] argue, varying $T$ is useful in order to understand the small-sample properties of the tests and the validity of asymptotic approximations. For instance, we elect to examine a 5 year window since this may show how distorted results from taking a really small sample could potentially be, and also it is a commonly adopted horizon when using rolling windows. A 30 year window corresponds approximately to the sample sizes of Fama and French [15, 16] and Jagannathan and Wang [27]; while the 600 month sample matches the largest sample examined by Jagannathan and Wang [28]. We also examine 1000 months since this approximates the current size of the largest sample available on the Kenneth French's library [January 1927 to December 2009 - 996 months], and could be considered as an approximation of the asymptotic variance. The estimators and specification tests are then calculated based on the $T$ samples of the factors and returns generated from the factor model. We repeat this independently to obtain 10,000 draws of the estimators of $\lambda, \pi$ (the pricing errors) and $J$ (the overidentifying restriction statistic).

Previous related empirical studies such as Kan and Zhou [35, 33], Jagannathan and Wang [28] and Cochrane [13] focus on the CAPM model to test the efficiency of Beta and SDF methods. In this sense, our contribution is to evaluate the methods on multifactor models in order to check for consistency in presence of other more leptokurtic factors commonly used by researchers. Therefore, we evaluate the two methods by estimating and testing the CAPM, Fama and French, RUH and the Carhart four factor models. We denote the factors as the excess market return (RMRF), size (SMB), value (HML) and momentum 
(UMD). ${ }^{6}$

In order to generate the excess returns from equation (4) we first need the $N \times K$ matrix $\beta$, capturing the sensitivity of returns to the factor(s). This $\beta$ matrix previously defined in equation (2), represents the slope coefficients in the OLS regressions of each $N$-test portfolio and $K$-factor model. We use three values of $N$ to generate $\beta$, these are the value weighted returns of the 10 size-sorted portfolios, the 25 Fama-French portfolios (the intersections of the 5 size and 5 book-to-market portfolios) and the 30 industry portfolios. As Lewellen, Nagel and Shanken [36] suggest, the traditional tests portfolios used in empirical work such as the size and 25 size/value sorted portfolios frequently present a strong factor structure, hence it seems reasonable to adopt other criteria (industry) for sorting.

In Table 10 we report the descriptive statistics of historical observations of factors and test portfolios, these values are used to calibrate the simulations. As can be seen from the four moments shown, the factors associated with the multi-factor models are quite different from the excess market return factor, in particular the momentum factor is almost three times more leptokurtic than the excess market return. Thus, it is important to consider an alternative which captures properties more consistent with the data such as excess kurtosis. Similar studies such as Kan and Zhou [34] consider the Student- $t$ distribution; however the magnitude of kurtosis is still limited for a $t$-distribution with a finite fourth moment. ${ }^{7}$ In previous simulations not showed here, a Student- $t$ distribution with five degrees of freedom implies a kurtosis of 6 for the RMRF factor, which is still much lower than the empirical value of 11 . Therefore, we consider the empirical distribution as the alternative to the multivariate normal.

\footnotetext{
${ }^{6}$ See Fama and French [16], for a complete description of these factors.

${ }^{7}$ The asymptotic distribution theory for the GMM requires that returns and factors have finite fourth moments. Hence, there must be more than four degrees of freedom.
} 


\section{Results}

We find that the choice of following either the Beta or the SDF method to empirically estimate and evaluate an asset pricing model can be traduced into a choice between efficiency or robustness. In particular, we show that frequently the Beta method dominates in terms of efficiency whereas the SDF method dominates in terms of robustness. Perhaps one of the most influential and cited papers in this field is the one of Jagannathan and Wang [28], they argue that both methods lead to estimates with similar precision even in finite samples. Here, we illustrate that the conclusion of Jagannathan and Wang [28] are only valid under very specific conditions, and cannot be generalized.

\subsection{Comparison of $\lambda$ estimators}

Tables 1 and 2 compare the performance of Beta and SDF methods at estimating $\lambda$ by the CAPM model using US data. Here, our results are qualitative and quantitative similar to those on Jagannathan and Wang [28]. In particular, Table 1 shows that the expected value and the standard error of $\widehat{\lambda}^{*}$ and $\widehat{\lambda}$ are quite similar. Actually, the standard error of Beta estimators is statistically equal to the standard error of SDF estimators in most of the cases. Then, under this specific framework there are virtually no differences in terms of efficiency between the Beta and SDF methods. One of the main implication of this results is that there are no significative advantages to applying the Beta method to nonlinear asset pricing models formerly expressed in SDF representation through linear approximations.

\section{[INSERT TABLE 1 HERE]}

In order to conduct a clearer comparison of the standard errors of $\lambda$ we present Table 2 . Table 1 allows us to compare $\sigma\left(\widehat{\lambda}^{*}\right)$ versus $\sigma(\widehat{\lambda})$ instead of $\sigma(\widehat{\delta})$ versus $\sigma(\widehat{\lambda})$ in order to avoid misleading conclusions driven by a scaling issue. However, if we consider the possibility of intrinsic differences among the methods, the expected values of $\widehat{\lambda}^{*}$ and $\widehat{\lambda}$ do not necessarily would be similar in general. For this reason it is convenient to compute ratios of relative 
standard errors such as $\sigma_{r}(\lambda)=\sigma(\lambda) \div \mathrm{E}(\lambda)$. By doing so, we would have an accurate measure of the relative efficiency of the methods.

\section{[INSERT TABLE 2 HERE]}

Ratios close to the unity represent a high degree of similarity of the efficiency of both methodologies at estimating $\lambda$. Higher ratios suggest that following the Beta method is better even in finite samples than following the SDF method to make inferences on $\lambda$ estimators. Given that one is generally interested in testing the null hypothesis that the estimator is statistically equal to zero, the ratios such as the ones in Table 2 offer a good indication of which method lead to more accurate inferences. In particular, all values of Table 2 are slightly greater than one, which mean that the Beta method lead to lower standard errors when estimating the CAPM. In general, the values of the ratios diminish as we increase the size of the sample, and second-stage SDF estimators are indeed more efficient than first-stage estimators as expected. In addition, the un-centred SDF specification reveal a marginal advantage compared with the centred specification probably due to the extra moment restriction.

Our original contribution regarding the comparison of $\sigma_{r}(\lambda)$ is on the evaluation of multifactor asset pricing models. Tables 3, 4 and 5 show the ratios of relative standard errors of the Beta and SDF methods for the Fama-French, RUH and Carhart models respectively. The correspondent expected values and standard errors for the multi-factor asset pricing models which were used in the construction of Tables 3,4 and 5 are on the appendix section in Tables 11, 12 and 13. We begin by describing the results for the Fama-French model, which is loaded with the market factor, the size factor and the value factor.

\section{[INSERT TABLE 3 HERE]}

The first (upper) panel of Table 3 is comparable to the Table 2 because in both cases the estimated $\lambda$ correspond to the market factor. Thus, it is not surprisingly to find a similar pattern which reinforce the conclusion that there are no significative differences 
when estimating the parameter associated with the market factor. The un-centred SDF method is again more efficient than the centred SDF method at estimating $\lambda$. And this time the second-stage un-centred method is marginally more efficient than the Beta method for samples smaller than one thousand.

Contrary to the market factor case, the standard error of Beta estimators linked to the size and value factors are statistically smaller than the correspondent standard errors of SDF estimators. This become evident in the higher ratios of second and third panels of Table 3. These results suggest that the empirical equivalence of both methods is subject to the loaded factor in the asset pricing model. In particular, the market factor do not represent a challenge to the SDF method, whilst the value factor can lead to significative differences according to the $\sigma_{r}(\lambda)$ ratios. For instance, the relative standard error of the un-centred first-stage SDF method can be more than twice as big than the relative standard error of the Beta method. Beta estimators are even more efficient than second-stage SDF estimators, which by construction are intended to increase the estimation efficiency of $\widehat{\lambda}$.

The second multi-factor asset pricing model is the RUH, which factors are market, momentum and value. The estimation of the RUH model allow us to compare the efficiency of the estimator associated with the market and value factors in previous tables and introduces the result for momentum. The ratios of relative standard errors for the RUH model are on Table 4.

\section{[INSERT TABLE 4 HERE]}

The ratios of relative standard errors $\sigma_{r}(\lambda)$ linked to the market factor are slightly lower than one; however the standard errors $\sigma(\lambda)$ are statistically equal for most of the cases. On the other hand, the magnitudes for the value factor are similar to the ones of the FamaFrench model in Table 3. The second panel of Table 4 show the ratios for the momentum factor, which are somewhat greater than the ratios for value factor. Here, we also find that SDF method may have difficulties in small samples which is reflected in values of 17.7 and 8.5 . 
The third and last multi-factor asset pricing model estimated is the Carhart model, which factors are market, size, value and momentum. The estimation of Fama-French, RUH and Carhart models represent the core contribution to the field, which lead to the main original results. However, we also present results for other test portfolios and to reduced sample sizes such as the ones researchers may face at evaluating models using UK data samples. For now, we show the relative standard errors of Carhart model $\lambda$ estimators in Table 5.

\section{[INSERT TABLE 5 HERE]}

The results for the Carhart model support the argument that the efficiency of the method is associated with the factor. The lower ratios of relative standard errors of $\lambda$ estimators are those linked to the size factor, followed by the market, value, and the greater are those of momentum factor. If the methods were empirically equal efficient, this ratios should be similar across panels. However this is not the case, apparently the relative efficiency is related with the associated factor of each estimator. Table 5, suggest that SDF method marginally dominates Beta method in first and second panels which correspond to the results associated with the market and size factors, whilst Beta method outperform SDF method for the third and fourth panels which are related with the value and momentum factors. In sum, the previous tables show that the relative efficiency of the methods are not - at least - independent of the factors.

One may argue that results on Tables 3,4 and 5 may be partially driven by the added factors, and not because any intrinsic difference among the methods. In order to address this possibility, we estimate four alternative single-asset pricing models. In each alternative, the model is loaded with a different factor namely market (CAPM), size, value and momentum. The ratios of relative standard errors of the Beta and SDF methods for these single-asset pricing models are on Table 6 . The correspondent expected values and standard errors are on the appendix section in Table 14 .

[INSERT TABLE 6 HERE] 
According to Table 6, Beta method lead to more accurate inferences than SDF method. The relevance of Table 6 is that we artificially fix every element in the evaluation of the asset pricing model except the loaded factor. Given that ratios across panels are different, the statistical characteristics of each factor, as well as their relation to the test portfolios, are presumably the main drivers behind the differences of the methods at estimating $\lambda$.

Since we do not impose any distributional assumption to the simulation of factors and test portfolios, we cannot derive an expression for the asymptotic variance of the $\lambda$ estimator, which could explicitly reveal what drives the efficiency of the estimator via comparative static analysis. However, we can still empirically reveal how the statistical characteristics of factors and their relation to the test portfolios relates to the variance of $\hat{\lambda}^{*}$ and $\widehat{\lambda}$. For this purpose, we set up an alternative simulation exercise based on the bootstrap generation of factors by using a restricted Pearson system, which has been proven to capture the complex behavior exhibited in financial data. This new simulation exercise is different from the one explained in subsection 2.4 which give rise to Tables 2, 3, 4 and 5 presented previously. In particular, we now simulate 200 series of size $T=996$ for the market, size, value and momentum factors. The particularity of this new factor simulation is that the mean, skewness and kurtosis are set to be less than $5 \%$ different than in the original historical series (see Table 10 for the descriptive statistics of the original samples of returns and factors). The new resulting simulated series have different variances but virtually identical mean, skewness and kurtosis, whilst the previous factors generated from the empirical distribution allow for greater variation in the mean, variance, skewness and kurtosis since we do not impose any restriction to the data-generating process. By fixing the first, third and fourth moments of the market, size, value and momentum factors, is to some extent analogous to partially derive the asymptotic variance of the estimator with respect to the factor variance.

Figures 1, 2, 6 and 7 show the results for US data, whilst Figures 3, 8, 9, and 10 show the results for UK data which size is $T=339$. For each of these Figures we plot the values of $\widehat{\lambda}^{*}$ and $\widehat{\lambda}_{1}^{A}$ with respect to the factor variance and the second moment of returns and 
factors. Top panels of these new set of Figures are for Beta estimators, bottom panels for SDF estimators, left panel for factor variance dependency, and right panel for second moment matrix of returns and factor dependency. The first distinguish feature comes from the comparison of top versus bottom panels, in which we can identify a significant greater variation of the SDF estimator with respect to the Beta estimator. We do not present the comparison of the centred SDF method since results are quite similar; however, they are available upon request. Figure 1 correspond to the results of the market factor of this new simulation.

\section{[INSERT FIGURE 1 HERE]}

The mean of the factor is equal to the $\delta$ estimator in the Beta method, so any fluctuation on $\widehat{\delta}$ is independent of the higher order factor moments. Therefore, the negative relation between $\widehat{\lambda}^{*}$ and the variance of the factor is straightforward explained by the definition of $\widehat{\lambda}^{*}$ in equation (19). In other words, the negative relation on the upper-left panel of Figure 1 is mainly driven by the transformation from $\widehat{\delta}$ to $\widehat{\lambda}^{*}$. On the other hand, the lower-left panel show that the values of $\widehat{\lambda}_{1}^{A}$ are extremely sensible to variations in the factor variance $\Omega$. This high sensitivity of $\sigma\left(\widehat{\lambda}_{1}^{A}\right)$ is present for low or high values of $\Omega$. In sum, the variance of the factor does not seem to have a significant role at explaining the fluctuations of Beta estimators since $\widehat{\delta}$ is independent of $\Omega$. However, the fluctuations of SDF estimators seem to be quite sensible to $\Omega$.

According to Table 10, the variance of the market factor for the US is around 30. A value of $\Omega \approx 30$ in Figure 1 may correspond to values of $\widehat{\lambda}_{1}^{A}$ between -200 to 300 . However, we know from Table 1 that the expected value of $\hat{\lambda}^{*}$ and $\widehat{\lambda}_{1}^{A}$ are around 1.8. The question that arises is why market factors with variance around 30 may lead to such dramatic fluctuations on $\widehat{\lambda}_{1}^{A}$ given that we know that the value of $\sigma\left(\widehat{\lambda}_{1}^{A}\right)$ is only 0.64 ? The answer rely on the high covariance, or the high second moment of returns and factor.

Right panels of Figure 1 relates the values of Beta and SDF $\lambda$ estimators to the second moment of returns and factor. As expected, Beta estimators are independent on the 
covariance of returns and factors. Contrary, SDF estimators reveal a clear relation with respect of the second moment of returns and factors. To better understand this relation we can refer to equation (15) in which we illustrate the moment conditions of the un-centred SDF method which is minimized by GMM. The equation (15) imply that $\mathrm{E}\left[r_{t}\right] \approx \lambda \mathrm{E}\left[r_{t} f_{t}\right]$ where $\mathrm{E}\left[r_{t} f_{t}\right]$ represent the second moment matrix of returns and factor. Thus, relative high values of $\mathrm{E}\left[r_{t} f_{t}\right]$ represent an advantage to the SDF method at estimating $\widehat{\lambda}_{1}^{A}$ with greater precision. By the same token, values of $\mathrm{E}\left[r_{t} f_{t}\right]$ close to zero would represent a drawback of the SDF method at estimating $\widehat{\lambda}_{1}^{A}$ because the method would allow all the necessary variation on the estimator in order to validate $\mathrm{E}\left[r_{t}\right] \approx \lambda \mathrm{E}\left[r_{t} f_{t}\right]$.

According to the historical series of market returns and the 10 size sorted portfolios, the value of $\mathrm{E}\left[r_{t} f_{t}\right]=37.19$. This value is identified in the black squared data cursor. The corresponding values of 2.008 and 2.189 are not identical to those on Table 1 since the values on Figure 1 correspond to one single estimation (using the original historical dataset) whereas results on Table 1 correspond to the expected value of 10,000 realizations.

This evidence reveal that results such as the ones presented on Jagannathan and Wang [28] which argue that there are no differences in the efficiency of the Beta and SDF methods, are not driven by intrinsic similarities of the methods. In fact, the similarity in terms of efficiency is driven by the relative high value of $\mathrm{E}\left[r_{t} f_{t}\right]$ present in the CAPM. In other words, if the Beta and SDF methods lead to similar levels of efficiency at estimating $\lambda$ can be mostly explained by the statistical properties of both the returns and factors rather than any empirical equivalence of the methods.

It is worthwhile to clarify that $\mathrm{E}\left[r_{t} f_{t}\right]$ is actually a $N \times K$ matrix, which for our case is a $10 \times 1$ vector. The values of $\mathrm{E}\left[r_{t} f_{t}\right]$ in Figure 1 are in fact the average of each vector values.

Figures 6 and 7 correspond to the size and value factors, and are located in the appendix. The values of $\mathrm{E}\left[r_{t} f_{t}\right]$ are 7.521 and 6.428 for size and value factors, which are lower than the correspondent value of $\mathrm{E}\left[r_{t} f_{t}\right]$ for the market factor of 37.19. Therefore, it is more likely that the SDF method would lead to less accurate $\lambda$ estimators. Graphically, the largest 
value of $\mathrm{E}\left[r_{t} f_{t}\right]$, the less volatile $\widehat{\lambda}$ should be, so that $\lambda \mathrm{E}\left[r_{t} f_{t}\right]$ would asymptotically converge to $\mathrm{E}\left[r_{t}\right]$, which has a value of 0.85 for the 10 size-sorted portfolios according to Table 10 . If the covariance is rather low, as shown in Figures 6 and 7 , the method would vary $\widehat{\lambda}$ in order to $\lambda \mathrm{E}\left[r_{t} f_{t}\right]$ could asymptotically converge to $\mathrm{E}\left[r_{t}\right]$, and this is traduced in high values of $\widehat{\lambda}$.

Figure 2 deserves a special attention since the momentum factor has a skewness equal to -3.03 , and this high and negative value has important implications in the comparison of both methods.

\section{[INSERT Figure 2 HERE]}

As far as the factor exhibits a large and negative skewness, the chances that the covariance of returns and factor become negative increases. A negative value of $\mathrm{E}\left[r_{t} f_{t}\right]$ would force the SDF method to deliver a negative $\lambda$ in order to validate $\mathrm{E}\left[r_{t}\right] \approx \lambda \mathrm{E}\left[r_{t} f_{t}\right]$ because in general $\mathrm{E}\left[r_{t}\right]>0$. This is less likely to happen in the Beta method since the value of the estimator basically depend on the first moment of the factor. Figure 2 show that the value of $\mathrm{E}\left[r_{t} f_{t}\right]$ for momentum factor is 10.83 , which is higher than those on size and value factor, but less than the correspondent of the market factor. For one side, the higher covariance with respect to the size and value factors tend to decreases the fluctuations of $\widehat{\lambda}$, but the marked negative skewness is the responsible of the negative value of the estimator, which is equal to -10.88 , and equal to 2.932 for the Beta method.

The size of the sample is another criteria in which we can test the performance of the Beta and SDF methods. Researchers and practitioners frequently faces with small samples at estimating and evaluating asset pricing models, and it is important to know in what extent the efficiency of the method is subject to small samples. In order to address this issue, we consider a different data set. In particular we take UK data from Alan Gregory library, which goes from October 1980 to December 2008, and a size of $T=339$. This is notably smaller than for the US data from Kenneth R. French library, which goes from January 1927 to December 2009, and the size of $T=996$. Figures 3, 8, 9, and 10 show the 
results for the UK data samples. In this section we present the Figure 3 with the results of UK size factor and we leave the rest for the appendix section.

[INSERT FIGURE 3 HERE]

Table 10 reveal that the UK size factor has a mean equal to -0.04 , a negative skewness and the average of the vector of the second moment of returns and factor is equal to -0.015 . Under this conditions, and according to our previous analysis, this imply high fluctuations on $\hat{\lambda}$, and Figure 3 corroborates it. It is uncommon that traded factors such as market, size, value and momentum have a negative, or close to zero mean value. However, this is one exemption, which increases the possibility that Beta estimators become negative. In particular $\widehat{\lambda}=-0.44$, and $\widehat{\lambda}^{*}=-7.06$

Our results show that the efficiency of SDF method represented by the value of $\sigma(\widehat{\lambda})$ is highly sensible to the low covariance of the returns and factors. Besides, the higher order moments of factors such a negative skewness may cause negative values of $\lambda$ which are at least not easily interpreted in economic terms. The second-stage estimators naturally increase the efficiency of the SDF method, however this gains are not enough to outperform the efficiency of Beta method. We could force the SDF method to deliver $\lambda$ estimators with greater accuracy by including its definition - see equation (19) - into the moment restrictions of the un-centred SDF method in equation (15), or into the centred moment restrictions of the SDF method in equation (17). Nevertheless, this alternative is not the way the SDF method is usually implemented, and our main interest is evaluating the Beta and SDF methods as existing approaches.

Results such as in Jagannathan and Wang [28] are conceivable because the covariance of the returns and factors is especially large for the market factor and because this particular factor is almost centred. However, we show that factors with a negative skewness, and a low covariance with respect to the returns such as momentum, entail significant differences in the estimation efficiency of $\lambda$ and $\delta$ estimators. 


\subsection{Results: Robustness}

In this subsection we turn our attention to the pricing errors estimates $\widehat{\pi}$, $\widehat{\pi}^{*}$, their corresponding standard errors $\sigma(\widehat{\pi}), \sigma\left(\widehat{\pi}^{*}\right)$ and their associated $\widehat{J}$, and $\widehat{J}^{*}$ statistics. The method would be more robust than the other if their simulated standard error of the pricing errors is lower.

To better understand where the advocated trade-off between efficiency and robustness do comes from, we need to briefly describe the set of moments for each method. The traditional Beta GMM restrictions incorporate three sets of moments: (1) the $N$ asset pricing restriction which define the $\alpha$ vector; (2) the $N \times K$ zero covariance between errors and factors; and (3) the $K$ definition of $\delta$, which is equal to the mean of the traded factor. Therefore, by imposing the definition of $\delta$, the Beta method increases their relative estimation efficiency. On the other hand, the SDF method only incorporates the $N$ asset pricing restriction for the un-centred specification which define $\pi$; and the $N$ asset pricing restriction plus the $K$ mean of the factor for the centred specification. However, this method does not impose the definition of $\lambda$. As a result, it allows for freely vary its parameter in order to achieve lower pricing errors, favoring the robustness rather than the efficiency. By the same token, the specification test in the Beta method tends to under-reject in finite samples while the SDF method has approximately the correct size.

\section{[INSERT TABLE 7 HERE]}

Table 7 show that multi-factor asset pricing models can better explain the cross-section of expected returns than the single asset pricing model. The evidence is on the lower expected values of the pricing errors as we move from the CAPM to the Fama-French, RUH and Carhart models. Also, the expected value of pricing errors for all models diminish as we move from smaller to larger sample sizes as expected. Now, regarding the comparison between the Beta and SDF methods, our results show that the SDF method does a better job at explaining more of the cross-section of expected returns than the Beta method. Here, we are able to identify that second-stage SDF lead to larger pricing errors than first-stage. As 
it happen with OLS and GLS, first-stage is devoted to minimize the pricing errors whereas second-stage is not. Therefore, it is not surprising that second-stage SDF estimators do not achieve lower pricing errors. Table 7 also allows us to compare the centred and un-centred SDF specifications. As we can derive from the table, the un-centred method leads to lower pricing errors than the centred method in general. This can be explained because of the added moment restrictions in the centred method.

\section{[INSERT TABLE 8 HERE]}

In order to conduct a comparison free from measuring bias, we compute relative standard errors in Table 8 in the same way as we did with the $\lambda$ estimators. Our results show that first-stage SDF method is more robust than Beta method; the reported ratios are in all cases lower than 1 , specifically goes from 0.57 to 0.85 . However, second-stage SDF method and Beta method are similar robust since ratios are all around 1, in particular they go from 1.1 to 0.89 . Our results reveal that the lower values of $\widehat{\pi}$ are those associated with the firststage un-centred SDF method. The reason is that this method is devoted to exclusively minimize the pricing errors as the weighting matrix in the GMM is the identity matrix. Any other SDF representation as the second-stage or centred SDF, would lead to increases in $\widehat{\pi}$ given that the added moment restrictions or due to a different weighting matrix in the GMM.

In retrospective, we show in subsection 3.1 that Beta method achieve more efficient $\hat{\lambda}$ estimators than SDF method. And that even the second-stage SDF method, which increases the efficiency of the estimator, was not as efficient as the Beta method. Here, the firststage SDF method is more robust than the Beta method. However, the robustness of the Beta method can be compared with the robustness of the second-stage SDF method. The bottom line is that no method fully dominates the other; in fact they are complementary and cannot be considered as empirically equivalent.

To examine the specification test in the two methods, we compute the rejection rates under the null hypothesis that the model holds. We report the test size for three significant 
levels: 1 percent, 5 percent and 10 percent. To estimate the tails of the sampling distribution of the $J$-statistics, we perform 10,000 simulations. To examine the standard deviation of $\widehat{\lambda}$ and $\widehat{\pi}$, there is no significant difference whether one performs 1,000 or 10,000 simulations since one takes the standard deviation of the series. However, when examining the tail of the $J$-statistic distribution, it is preferable to perform 10,000 instead of 1,000 simulations. This approach is also adopted in Shanken and Zhou [44] and Kan and Zhou [33] among others.

\section{[INSERT TABLE 9 HERE]}

In Table 9 we present the results for the specification tests, for brevity, we show only the results from the 10 size-sorted portfolios for the CAPM and in Tables 17, 18 and 19 the corresponding values for the Fama-French, RUH and Carhart models respectively. Contrary

to the case of the analysis of $\widehat{\lambda}$ and $\widehat{\pi}$, the specifications tests do not yield significant differences when comparing the single and multi-factor cases. Therefore, in general, the conclusions reached by Table 9 can be extended to those in Tables 17, 18 and 19. We find that most tests have better finite sample performances for a smaller number of assets, this is consistent with related works for Beta pricing model comparisons such as in $\mathrm{Li}, \mathrm{Xu}$ and Zhang [37].

The specification tests represent rejection rates of the $J$-statistic. Nevertheless, it is well known that this statistic can be computed in many ways, for instance, depending on the choice of the weighting matrix used in the aggregation of the pricing errors. We use three alternatives to give a more robust idea about the performance of the methods. In particular we use the spectral density matrix $W=S$, the second moment matrix $W=\mathrm{E}\left[r_{t} r_{t}^{\prime}\right]$, and the covariance matrix of returns $W=\operatorname{Cov}\left[r_{t}\right]$.

To add the pricing error vector $g_{T}(\theta)$, the $J$-statistic, is weighted by the covariance of the pricing errors in the first-stage, which is simultaneously weighted by the spectral density matrix $S$, equation (10). Therefore, both the first and second-stage $J$-statistics are weighted by $S$ in order to aggregate the pricing errors vector $g_{T}(\theta)$. As we know from Hansen [21], 
this choice is statistically optimal in the sense that it maximizes the asymptotic statistical information in the sample about a model, given the choice of moments. However, since the $S$ matrix changes across models, it is not convenient to use it for model comparison. In particular, we could not claim a better fit because of a smaller $J$-statistic since we have different values of $S$ across models.

Hansen and Jagannathan [22] suggest the use of the second moment matrix of excess returns $W=\mathrm{E}\left[r_{t} r_{t}^{\prime}\right]$ instead of $W=S$. This alternative is more suitable for model comparison because it is invariant to the model and their parameters. It provides an economic measure of the model fit instead of a statistical measure and has the property of being invariant to portfolio formation. For our third alternative we follow Burnside [7], Balduzzi and Yao [3] and Kan and Robotti [31] who suggest that the centred SDF method should use the covariance matrix of excess returns $W=\operatorname{Cov}\left[r_{t}\right]$.

Tables 9, 17, 18 and 19, illustrate that the Beta method consistently under-rejects in finite-samples. The level of under-rejection is considerably more for the multi-factor models where in some cases the size is only about $1 \%$ of the theoretical value. Interestingly, in the case of the Beta method, the $J$-statistic leads to the same results when we adopt $W=S$.

In contrast to performance of the Beta method, the size of the SDF method is much closer to the theoretical values regardless of the model. In fact, this is partially explained in Table 7 since a more efficient pricing error should lead to better specification tests in general. With respect to the differences in size between the two SDF specifications, we see that there is a marginal increase in size for centred SDF with respect to un-centred SDF. 


\section{Conclusion}

The interest on learning about the asymptotic and finite sample properties of asset pricing model estimators, like risk premiums and pricing errors, have attracted the attention of researchers for decades. This interest is motivated for an extensive list of theoretical and empirical applications mainly - but not exclusive - in economics and finance areas. It is not uncommon to find examples in which different econometric approaches involves a trade-off between efficiency and robustness since more efficient estimators may usually comes at the cost of higher pricing errors and vice versa. However, as far as to our knowledge, this is the first time in which such analogy is explicitly used to better understand the differences of the Beta and SDF methods. This evidence is useful for researchers and practitioners because they could choose a proper procedure in terms of a given application. Consequently, the adequate selection leads to more accurate hypothesis tests, and other kind of computations.

We argue that current studies which compare both approaches are conducted under certain conditions that are not sufficient to differentiate their performance. Once we relax those conditions, we show that differences between the two methods unsurprisingly emerge. Specifically, we find evidence suggesting that the Beta method lead to better risk premium estimators while the SDF method lead to better pricing error estimators in terms of efficiency. We evaluate the magnitude of the resulting biases and we also illustrate what are the main elements that drive such differences.

The debate about the studied methods is still open. Presumably, given the response of Jagannathan and Wang [28] to Kan and Zhou [35], the dominant trend is to consider that the methods do not exhibit significant differences in terms of efficiency. For example, see Wang and Zhang [49], Jagannathan, Skoulakis and Wang [26], Vassalou, Li and Xing [47], Cochrane ([12], [13]), Smith and Wickens [46], Nieto and Rodrguez [43], Balvers and Huang [4], Brandt and Chapman [6], and Cai and Hong [8], just to mention a few. Our results suggest that this similarity between the two methods only holds under very specific conditions. Once the asset pricing model under consideration includes more factors with 
greater non-normalities, the differences in terms of efficiency and robustness clearly emerge. In this sense, our results are closer to those on Kan and Zhou ([35],[33]) and Lozano and Rubio [39].

The purpose of this work is to contribute to the understanding of the finite sample properties of the Beta and SDF methods by showing evidence about the elements which determines the parameters bias. Further extensions to this work could be to explore what happen when considering non-traded factors. In principle, there is no reason to expect a similar pattern. For example, Kan and Robotti [31] show that the standard errors under correctly specified and potentially mispecified models are similar for traded factors, while they can differ substantially for non-traded factors such as the scaled market return and the lagged state variable CAY. Other kind of extensions would include the impact over other more specific areas in finance which traditionally follow one specific method. 


\section{References}

[1] Seung C. Ahn and Christopher Gadarowski, Small sample properties of the model specification test based on the hansen-jagannathan distance, Journal of Empirical Finance 11(1) (2004), 109-132.

[2] Christine E. Amsler and Peter Schmidt, A monte carlo investigation of the accuracy of multivariate capm tests, Journal of Financial Economics 14(3) (1985), 359-375.

[3] Perluigi Balduzzi and Tong Yao, Testing heterogeneous-agent models: An alternative aggregation approach, Journal of Monetary Economics 54(2) (2007), 369-412.

[4] Ronald J. Balvers and Dayong Huang, Productivity-based asset pricing: Theory and evidence, Journal of Financial Economics 86(2) (2007), 405-445.

[5] Fischer Black, Michael Jensen, and Myron S. Scholes, The capital asset pricing model: Some empirical tests, Studies in Theory of Capital Markets. Jensen, Ed. New York: Preager Publishers. 17 (1972), 79-121.

[6] Michael W. Brandt and David A. Chapman, Linear approximations and tests of conditional pricing models, National Bureau of Economic Research Working Papers 12513, (2006).

[7] Craig Burnside, Empirical asset pricing and statistical power in the presence of weak risk factors, Duke University and NBER Working Papers with number 13357 (2007).

[8] Zongwu Cai and Yongmiao Hong, Some recent developments in nonparametric finance, Advances in Econometrics 25 (2009), 379-432.

[9] John Y. Campbell, Andrew W. Lo, and A. Craig MacKinlay, The econometrics of financial markets, Princeton NJ: Princeton University Press, 1997.

[10] Mark M. Carhart, On persistence in mutual fund performance, Journal of Finance 52(1) (1997), 57-82. 
[11] Robert Chen and Raymond Kan, Finite sample analysis of two-pass cross-sectional regressions, Working Paper, University of Toronto, (2004).

[12] John H. Cochrane, A resurrection of the stochastic discount factor / gmm methodology, Working Paper, Graduate School of Business, University of Chicago, (2000).

[13] _ Asset pricing - revised edition, Princeton University Press, 2005.

[14] Philip H. Dybvig and Jonathan E. Ingersoll, Mean variance theory in complete markets, Journal of Business 55(2) (1982), 233-251.

[15] Eugene F. Fama and Kenneth R. French, The cross-section of expected stock returns, Journal of Finance 47(2) (1992), 427-465.

[16] _ Common risk factors in the returns on stocks and bonds, Journal of Financial Economics 33(1) (1993), 3-56.

[17] Eugene F. Fama and James D. MacBeth, Risk return and equilibrium: empirical tests, Journal of Financial Political Economy 81(3) (1973), 607-636.

[18] Heber Farnsworth, Wayne Ferson, David Jackson, and Steven Todd, Performance evaluation with stochastic discount factors, Journal of Business 75(3) (2002), 473503.

[19] Wayne E. Ferson, Tests of multifactor pricing models, volatility bounds and portfolio performance, Handbook of the Economics of Finance. Elsevier Science Publishers, North Holland, 2005.

[20] Wayne E. Ferson and Ravi Jagannathan, Econometric evaluation of asset pricing models, G. S. Maddala and C. R. Rao, Eds. Handbook of Statistics, Vol. 14: Statistical Methods in Finance. Elsevier, 1996.

[21] Lars Peter Hansen, Large sample properties of generalized method of moments estimators, Econometrica 50(4) (1982), 1029-1054. 
[22] Lars Peter Hansen and Ravi Jagannathan, Assessing specification errors in stochastic discount factor models, Journal of Finance 55(2) (1997), 557-590.

[23] Lars Peter Hansen and Scott F. Richard, The role of conditioning information in deducing testable, Econometrica 55(3) (1987), 587-613.

[24] Jonathan E. Ingersoll, Theory of financial decision making, Rowman and Littlefield Publishing, Inc., 1987.

[25] Ravi Jagannathan, Georgios Skoulakis, and Zhenyu Wang, Generalized method of moments: applications in finance, Journal of Business and Economics Statistics 20(4) (2002), 470-481.

[26] The analysis of the cross section of security returns, Handbook of Financial Econometrics. Edited By Yacine Ait-Sahalia and Lars Hansen 1 (2008), ch. 14.

[27] Ravi Jagannathan and Zhenyu Wang, The conditional capm and the cross-section of expected returns, Journal of Finance 51(1) (1996), 3-53.

[28] _ Empirical evaluation of asset-pricing models: A comparison of the sdf and beta methods, Journal of Finance 57(5) (2002), 2337-2367.

[29] Christian Julliard and Jonathan A. Parker, Consumption risk and the cross-section of expected returns, Journal of Political Economy 113(1) (2005).

[30] Raymond Kan and Cesare Robotti, A note on the estimation of asset pricing models using simple regression betas, Federal Reserve Bank of Atlanta in its series Working Paper with number 2009-12.

[31]__ Specification tests of asset pricing models using excess returns, Journal of Empirical Finance 15(5) (2008), 816-838.

[32] Raymond Kan, Cesare Robotti, and Jay Shanken, Pricing model performance and the two-pass cross-sectional regression methodology, Federal Reserve Bank of Atlanta in its series Working Paper with number 2009-11. 
[33] Raymond Kan and Guofu Zhou, Empirical asset pricing: the beta method versus the stochastic discount factor method, Working Paper, University of Toronto and Washington University in St. Louis, (2002).

[34] _ Modeling non-normality using multivariate t: Implications for asset pricing, Working Paper, (2003).

[35] _ A critique of the stochastic discount factor methodology, Journal of Finance 54(4) (1999), 1221-1248.

[36] Jonathan Lewellen, Stefan Nagel, and Jay Shanken, A skeptical appraisal of asset pricing tests, Journal of Financial Economics Forthcoming (2009) (2009), 175194.

[37] Haitao Li, Yuewu Xu, and Xiaoyan Zhang, Evaluating asset pricing models using the second hansen-jagannathan distance, Journal of Financial Economics Forthcoming (2009).

[38] John Lintner, Security prices, risk and maximal gains from diversification, Journal of Finance 20(4) (1965), 587-615.

[39] Martin Lozano and Gonzalo Rubio, Evaluating alternative methods for testing asset pricing models with historical data, Journal of Empirical Finance Forthcoming (2010).

[40] A. Craig MacKinlay and Matthew P. Richardson, Using generalized method of moments to test mean-variance efficiency, Journal of Finance 46(2) (1991), 511-527.

[41] Jan Mossin, Equilibrium in a capital asset market, Econometrica 34(4) (1966), 768783.

[42] Francisco Pe naranda and Enrique Sentana, A unifying approach to the empirical evaluation of asset pricing models, CEPR Discussion Paper no. 7943. London, Centre for Economic Policy Research. 
[43] Belén Nieto and Rosa Rodríguez, Modelos de valoración de activos condicionales: un panorama comparativo, Investigaciones Económicas XXIX (1) (2005), 33-72.

[44] Jay Shanken and Guofu Zhou, Estimating and testing beta pricing models: Alternative method and their performance in simulations, Journal of Financial Economics 84(1) (2007), 40-86.

[45] William F. Sharpe, Capital asset prices: A theory of market equilibrium under conditions of risk, Journal of Finance 19(3) (1964), 425-442.

[46] Peter Smith and Michael Wickens, Asset pricing with observable stochastic discount factors, Journal of Economic Surveys 16(3) (2002), 397-446.

[47] Maria Vassalou, Qing Li, and Yuhang Xing, Sector investment growth rates and the cross-section of equity returns, Journal of Business 79(3) (2006), 1637-1636.

[48] Raja Velu and Guofu Zhou, Testing multi-beta asset pricing models, Journal of Empirical Finance 6(3) (1999), 219-241.

[49] Zhenyu Wang and Xiaoyan Zhang, Empirical evaluation of asset pricing models: Arbitrage and pricing errors over contingent claims, Working Paper, Federal Reserve Bank of New York in its series Staff Reports with number 265.

[50] Motohiro Yogo, A consumption-based explanation of expected stock returns, The Journal of Finance 61(2) (2006), 539-580. 


\section{$5 \quad$ Tables \& Figures}

Table 1: Expected VAlue AND STANDARD ERrors For CAPM MOdel: US DATA, 10 SIZE-SORTED PORTFOLIOS.

The table presents the expected value and the standard error of $\lambda$ GMM estimates under the Beta and the SDF methods. The returns and factors are generated under the null hypothesis with the factors sampled from the empirical distribution. The first estimator decorated with * are from the Beta method; the second and third correspond to the first and second-stage un-centred SDF method; and the fourth and fifth to the first and second-stage centred SDF method. The results are presented for different lengths of time series observations, and they are based on 10,000 simulations.

\begin{tabular}{lcccccccccc}
\hline \hline$T$ & $\mathrm{E}\left[\widehat{\lambda}^{*}\right]$ & $\mathrm{E}\left[\widehat{\lambda}_{1}^{A}\right]$ & $\mathrm{E}\left[\widehat{\lambda}_{2}^{A}\right]$ & $\mathrm{E}\left[\widehat{\lambda}_{1}^{B}\right]$ & $\mathrm{E}\left[\widehat{\lambda}_{2}^{B}\right]$ & $\sigma\left(\widehat{\lambda}^{*}\right)$ & $\sigma\left(\widehat{\lambda}_{1}^{A}\right)$ & $\sigma\left(\widehat{\lambda}_{2}^{A}\right)$ & $\sigma\left(\widehat{\lambda}_{1}^{B}\right)$ & $\sigma\left(\widehat{\lambda}_{2}^{B}\right)$ \\
\hline 60 & 2.14 & 2.18 & 2.89 & 2.34 & 2.72 & 2.62 & 2.86 & 3.55 & 3.15 & 3.49 \\
360 & 1.93 & 1.94 & 2.04 & 1.98 & 2.03 & 1.00 & 1.07 & 1.06 & 1.11 & 1.07 \\
600 & 1.93 & 1.93 & 1.99 & 1.96 & 1.99 & 0.77 & 0.83 & 0.80 & 0.86 & 0.82 \\
1000 & 1.92 & 1.92 & 1.95 & 1.95 & 1.96 & 0.59 & 0.64 & 0.61 & 0.66 & 0.62 \\
\hline \hline
\end{tabular}


Table 2: Relative STANDARD ERRORS FOR CAPM MOdel: US DATA, 10 SIZE-SORTED PORTFOLIOS.

The table presents the relative standard errors of $\lambda$ GMM estimates under the Beta and the SDF methods, computed as $\sigma_{r}(\widehat{\lambda})=\sigma(\widehat{\lambda}) \div \mathrm{E}(\widehat{\lambda})$. The returns and factors are generated under the null hypothesis with the factors sampled from the empirical distribution. Estimators decorated with * are from the Beta method; with A and B to the un-centred and centred SDF method; and with 1 and 2 to the first and second-stage respectively. The results are presented for different lengths of time series observations, and they are based on 10,000 simulations.

\begin{tabular}{ccccc}
\hline \hline$T$ & $\sigma_{r}\left(\widehat{\lambda}_{1}^{A}\right) \div \sigma_{r}\left(\widehat{\lambda}^{*}\right)$ & $\sigma_{r}\left(\widehat{\lambda}_{2}^{A}\right) \div \sigma_{r}\left(\widehat{\lambda}^{*}\right)$ & $\sigma_{r}\left(\widehat{\lambda}_{1}^{B}\right) \div \sigma_{r}\left(\widehat{\lambda}^{*}\right)$ & $\sigma_{r}\left(\widehat{\lambda}_{2}^{B}\right) \div \sigma_{r}\left(\widehat{\lambda}^{*}\right)$ \\
\hline 60 & 1.0705 & 1.0024 & 1.0987 & 1.0476 \\
360 & 1.0720 & 1.0056 & 1.0938 & 1.0272 \\
600 & 1.0713 & 1.0029 & 1.0931 & 1.0248 \\
1000 & 1.0701 & 1.0023 & 1.0918 & 1.0239 \\
\hline \hline
\end{tabular}


Table 3: Relative Standard errors For FAma-French MOdel: US DATA, 10 SizeSORTED PORTFOLIOS.

The table presents the relative standard errors of $\lambda$ GMM estimates under the Beta and the SDF methods, computed as $\sigma_{r}(\widehat{\lambda})=\sigma(\widehat{\lambda}) \div \mathrm{E}(\widehat{\lambda})$. The returns and factors are generated under the null hypothesis with the factors sampled from the empirical distribution. Estimators decorated with * are from the Beta method; with A and B to the un-centred and centred SDF method; and with 1 and 2 to the first and second-stage respectively. The results are presented for different lengths of time series observations, and they are based on 10,000 simulations.

\begin{tabular}{lcccc}
\hline \hline$T$ & $\sigma_{r}\left(\widehat{\lambda}_{1}^{A}\right) \div \sigma_{r}\left(\widehat{\lambda}^{*}\right)$ & $\sigma_{r}\left(\widehat{\lambda}_{2}^{A}\right) \div \sigma_{r}\left(\widehat{\lambda}^{*}\right)$ & $\sigma_{r}\left(\widehat{\lambda}_{1}^{B}\right) \div \sigma_{r}\left(\widehat{\lambda}^{*}\right)$ & $\sigma_{r}\left(\widehat{\lambda}_{2}^{B}\right) \div \sigma_{r}\left(\widehat{\lambda}^{*}\right)$ \\
\hline 60 & 0.9723 & 0.9505 & 0.9860 & 0.9797 \\
360 & 1.0127 & 0.9996 & 1.0301 & 1.0177 \\
600 & 1.0138 & 0.9996 & 1.0321 & 1.0195 \\
1000 & 1.0208 & 1.0026 & 1.0377 & 1.0215 \\
\hline & & & Sizket \\
\hline 60 & 0.8059 & 0.7472 & 0.6759 & 0.6447 \\
360 & 1.1631 & 1.0365 & 1.1296 & 1.0054 \\
600 & 1.1926 & 1.0600 & 1.1709 & 1.0407 \\
1000 & 1.2218 & 1.0891 & 1.2069 & 1.0773 \\
\hline 60 & & & Value & \\
360 & 3.1809 & 2.3172 & 3.2675 & 2.4899 \\
600 & 3.2061 & 2.3086 & 3.2679 & 2.4175 \\
1000 & 3.2520 & 2.3804 & 3.2996 & 2.4548 \\
\hline \hline
\end{tabular}


Table 4: Relative STANDARD ERRORS FOR RUH MODEL: US DATA, 10 Size-SORTED PORTFOLIOS.

The table presents the relative standard errors of $\lambda$ GMM estimates under the Beta and the SDF methods, computed as $\sigma_{r}(\widehat{\lambda})=\sigma(\widehat{\lambda}) \div \mathrm{E}(\widehat{\lambda})$. The returns and factors are generated under the null hypothesis with the factors sampled from the empirical distribution. Estimators decorated with * are from the Beta method; with A and B to the un-centred and centred SDF method; and with 1 and 2 to the first and second-stage respectively. The results are presented for different lengths of time series observations, and they are based on 10,000 simulations.

\begin{tabular}{lcccc}
\hline \hline$T$ & $\sigma_{r}\left(\widehat{\lambda}_{1}^{A}\right) \div \sigma_{r}\left(\widehat{\lambda}^{*}\right)$ & $\sigma_{r}\left(\widehat{\lambda}_{2}^{A}\right) \div \sigma_{r}\left(\widehat{\lambda}^{*}\right)$ & $\sigma_{r}\left(\widehat{\lambda}_{1}^{B}\right) \div \sigma_{r}\left(\widehat{\lambda}^{*}\right)$ & $\sigma_{r}\left(\widehat{\lambda}_{2}^{B}\right) \div \sigma_{r}\left(\widehat{\lambda}^{*}\right)$ \\
\hline 60 & 0.9152 & 0.8797 & 0.9031 & 0.8843 \\
360 & 0.9757 & 0.9630 & 0.9809 & 0.9722 \\
600 & 0.9771 & 0.9664 & 0.9871 & 0.9793 \\
1000 & 0.9750 & 0.9688 & 0.9870 & 0.9825 \\
\hline & & \multicolumn{4}{c}{ Momentum } \\
\hline 60 & 2.4552 & 1.8284 & 17.7187 & 8.5634 \\
360 & 2.8891 & 2.4292 & 4.1523 & 3.3299 \\
600 & 2.9046 & 2.4203 & 3.5697 & 2.8999 \\
1000 & 2.9386 & 2.4895 & 3.3247 & 2.7787 \\
\hline 60 & 1.7444 & 1.2396 & 3.6298 & 2.8698 \\
360 & 2.6694 & 2.0098 & 3.1465 & 2.3586 \\
600 & 2.7557 & 2.0545 & 3.0618 & 2.2790 \\
1000 & 2.8546 & 2.1291 & 3.0546 & 2.2835 \\
\hline \hline
\end{tabular}


Table 5: Relative standard errors for Carhart Model: US Data, 10 Size-Sorted PORTFOLIOS.

The table presents the relative standard errors of $\lambda$ GMM estimates under the Beta and the SDF methods, computed as $\sigma_{r}(\widehat{\lambda})=\sigma(\widehat{\lambda}) \div \mathrm{E}(\widehat{\lambda})$. The returns and factors are generated under the null hypothesis with the factors sampled from the empirical distribution. Estimators decorated with * are from the Beta method; with A and B to the un-centred and centred SDF method; and with 1 and 2 to the first and second-stage respectively. The results are presented for different lengths of time series observations, and they are based on $\underline{10,000 \text { simulations. }}$

\begin{tabular}{|c|c|c|c|c|}
\hline$T$ & $\sigma_{r}\left(\widehat{\lambda}_{1}^{A}\right) \div \sigma_{r}\left(\widehat{\lambda}^{*}\right)$ & $\sigma_{r}\left(\widehat{\lambda}_{2}^{A}\right) \div \sigma_{r}\left(\widehat{\lambda}^{*}\right)$ & $\sigma_{r}\left(\widehat{\lambda}_{1}^{B}\right) \div \sigma_{r}\left(\widehat{\lambda}^{*}\right)$ & $\sigma_{r}\left(\widehat{\lambda}_{2}^{B}\right) \div \sigma_{r}\left(\widehat{\lambda}^{*}\right)$ \\
\hline \multicolumn{5}{|c|}{ Market } \\
\hline 60 & 0.8908 & 0.8807 & 0.8736 & 0.8944 \\
\hline 360 & 0.9494 & 0.9422 & 0.9565 & 0.9529 \\
\hline 600 & 0.9537 & 0.9459 & 0.9656 & 0.9601 \\
\hline 1000 & 0.9530 & 0.9473 & 0.9676 & 0.9628 \\
\hline \multicolumn{5}{|c|}{ Size } \\
\hline 60 & 0.0054 & 0.0051 & 0.0041 & 0.0042 \\
\hline 360 & 0.7916 & 0.7310 & 0.7200 & 0.6907 \\
\hline 600 & 0.8782 & 0.8078 & 0.8253 & 0.7781 \\
\hline 1000 & 0.9067 & 0.8338 & 0.8717 & 0.8147 \\
\hline \multicolumn{5}{|c|}{ Value } \\
\hline 60 & 1.5673 & 1.3304 & 3.4290 & 2.6993 \\
\hline 360 & 2.5558 & 2.1614 & 3.1455 & 2.4990 \\
\hline 600 & 2.6442 & 2.2286 & 2.9943 & 2.4368 \\
\hline 1000 & 2.7897 & 2.3610 & 3.0218 & 2.5053 \\
\hline \multicolumn{5}{|c|}{ Momentum } \\
\hline 60 & 2.3715 & 1.9220 & 10.7372 & 7.5424 \\
\hline 360 & 2.6740 & 2.3712 & 3.5054 & 3.1166 \\
\hline 600 & 2.7843 & 2.4844 & 3.2370 & 2.8996 \\
\hline 1000 & 2.8425 & 2.5227 & 3.1375 & 2.7829 \\
\hline
\end{tabular}


Table 6: RElative STANDARD ERRORS FOR FOUR ALTERNATIVE SINGLE-FACTOR MODELS: US DATA, 10 SIZE-SORTED PORTFOLIOS.

The table presents the relative standard errors of $\lambda$ GMM estimates under the Beta and the SDF methods, computed as $\sigma_{r}(\widehat{\lambda})=\sigma(\widehat{\lambda}) \div \mathrm{E}(\widehat{\lambda})$. The returns and factors are generated under the null hypothesis with the factors sampled from the empirical distribution. Estimators decorated with * are from the Beta method; with A and B to the un-centred and centred SDF method; and with 1 and 2 to the first and second-stage respectively. The results are presented for different lengths of time series observations, and they are based on $\underline{10,000 \text { simulations. }}$

\begin{tabular}{|c|c|c|c|c|}
\hline$T$ & $\sigma_{r}\left(\widehat{\lambda}_{1}^{A}\right) \div \sigma_{r}\left(\widehat{\lambda}^{*}\right)$ & $\sigma_{r}\left(\widehat{\lambda}_{2}^{A}\right) \div \sigma_{r}\left(\widehat{\lambda}^{*}\right)$ & $\sigma_{r}\left(\widehat{\lambda}_{1}^{B}\right) \div \sigma_{r}\left(\widehat{\lambda}^{*}\right)$ & $\sigma_{r}\left(\widehat{\lambda}_{2}^{B}\right) \div \sigma_{r}\left(\widehat{\lambda}^{*}\right)$ \\
\hline \multicolumn{5}{|c|}{ Single-factor model loaded with market factor } \\
\hline 60 & 1.0705 & 1.0024 & 1.0987 & 1.0476 \\
\hline 360 & 1.0720 & 1.0056 & 1.0938 & 1.0272 \\
\hline 600 & 1.0713 & 1.0029 & 1.0931 & 1.0248 \\
\hline 1000 & 1.0701 & 1.0023 & 1.0918 & 1.0239 \\
\hline \multicolumn{5}{|c|}{ Single-factor model loaded with size factor } \\
\hline 60 & 1.7476 & 1.1466 & 1.7208 & 1.1851 \\
\hline 360 & 1.6270 & 1.0657 & 1.6295 & 1.0772 \\
\hline 600 & 1.6295 & 1.0654 & 1.6343 & 1.0757 \\
\hline 1000 & 1.6611 & 1.0707 & 1.6682 & 1.0802 \\
\hline \multicolumn{5}{|c|}{ Single-factor model loaded with value factor } \\
\hline 60 & 3.7779 & 1.8321 & 3.4502 & 2.7862 \\
\hline 360 & 3.1294 & 1.9587 & 3.1126 & 2.1024 \\
\hline 600 & 3.0371 & 1.9937 & 3.0441 & 2.0886 \\
\hline 1000 & 3.0626 & 1.9619 & 3.0809 & 2.0280 \\
\hline \multicolumn{5}{|c|}{ Single-factor model loaded with momentum factor } \\
\hline 60 & 3.0286 & 1.5298 & 3.0363 & 2.5698 \\
\hline 360 & 1.8116 & 1.4979 & 1.8630 & 1.6332 \\
\hline 600 & 1.7630 & 1.5079 & 1.8088 & 1.5939 \\
\hline 1000 & 1.7312 & 1.5073 & 1.7744 & 1.5683 \\
\hline
\end{tabular}


5 - Tables \& Figures

Table 7: EXPECTED VALUE AND STANDARD ERRORS FOR FOUR ALTERNATIVE ASSET PRICING MODELS: US DATA, 10 SIZE-SORTED PORTFOLIOS.

The table presents the expected value and the standard error of $\pi$ GMM estimates under the Beta and the SDF methods. The returns and factors are generated under the null hypothesis with the factors sampled from the empirical distribution. The first estimator decorated with * are from the Beta method; the second and third correspond to the first and second-stage un-centred SDF method; and the fourth and fifth to the first and secondstage centred SDF method. The results are presented for different lengths of time series observations, and they are based on 10,000 simulations.

\begin{tabular}{|c|c|c|c|c|c|c|c|c|c|c|}
\hline$T$ & $\mathrm{E}\left[\widehat{\pi}^{*}\right]$ & $\mathrm{E}\left[\widehat{\pi}_{1}^{A}\right]$ & $\mathrm{E}\left[\widehat{\pi}_{2}^{A}\right]$ & $\mathrm{E}\left[\widehat{\pi}_{1}^{B}\right]$ & $\mathrm{E}\left[\widehat{\pi}_{2}^{B}\right]$ & $\sigma\left(\widehat{\pi}^{*}\right)$ & $\sigma\left(\widehat{\pi}_{1}^{A}\right)$ & $\sigma\left(\widehat{\pi}_{2}^{A}\right)$ & $\sigma\left(\widehat{\pi}_{1}^{B}\right)$ & $\sigma\left(\widehat{\pi}_{2}^{B}\right)$ \\
\hline & \multicolumn{10}{|c|}{ CAPM } \\
\hline 60 & 36.57 & 24.20 & 47.46 & 24.94 & 41.84 & 20.68 & 11.28 & 30.43 & 11.61 & 25.02 \\
\hline 360 & 15.14 & 10.03 & 15.56 & 10.17 & 15.45 & 8.63 & 4.70 & 8.92 & 4.77 & 8.80 \\
\hline 600 & 11.69 & 7.75 & 11.82 & 7.85 & 11.83 & 6.65 & 3.62 & 6.71 & 3.67 & 6.71 \\
\hline \multirow[t]{2}{*}{1000} & 9.08 & 6.01 & 9.09 & 6.09 & 9.15 & 5.19 & 2.82 & 5.16 & 2.86 & 5.20 \\
\hline & \multicolumn{10}{|c|}{ Fama-French } \\
\hline 60 & 24.99 & 11.92 & 30.82 & 12.86 & 27.47 & 12.78 & 3.84 & 21.47 & 4.17 & 17.72 \\
\hline 360 & 10.48 & 4.87 & 7.58 & 5.04 & 7.25 & 4.62 & 1.49 & 3.45 & 1.55 & 3.00 \\
\hline 600 & 8.51 & 3.76 & 5.41 & 3.87 & 5.30 & 3.68 & 1.14 & 2.22 & 1.17 & 2.05 \\
\hline \multirow[t]{2}{*}{1000} & 7.19 & 2.90 & 4.00 & 2.99 & 3.98 & 2.90 & 0.87 & 1.56 & 0.90 & 1.51 \\
\hline & \multicolumn{10}{|c|}{ RUH } \\
\hline 60 & 38.15 & 13.05 & 37.95 & 14.20 & 37.52 & 21.36 & 4.68 & 25.89 & 5.11 & 25.13 \\
\hline 360 & 15.89 & 5.24 & 11.26 & 5.49 & 11.44 & 8.57 & 1.70 & 6.28 & 1.78 & 6.20 \\
\hline 600 & 12.80 & 4.10 & 8.67 & 4.28 & 8.89 & 6.87 & 1.32 & 4.69 & 1.38 & 4.72 \\
\hline \multirow[t]{2}{*}{1000} & 10.48 & 3.15 & 6.51 & 3.29 & 6.73 & 5.52 & 1.01 & 3.39 & 1.05 & 3.47 \\
\hline & \multicolumn{10}{|c|}{ Carhart } \\
\hline 60 & 34.58 & 10.43 & 27.92 & 11.59 & 27.64 & 20.28 & 3.53 & 19.84 & 3.93 & 18.75 \\
\hline 360 & 17.19 & 4.36 & 7.01 & 4.60 & 7.00 & 8.45 & 1.42 & 3.38 & 1.50 & 3.15 \\
\hline 600 & 15.20 & 3.37 & 5.00 & 3.54 & 5.07 & 6.62 & 1.08 & 2.17 & 1.14 & 2.11 \\
\hline 1000 & 13.85 & 2.62 & 3.69 & 2.74 & 3.77 & 5.26 & 0.85 & 1.53 & 0.89 & 1.53 \\
\hline
\end{tabular}


Table 8: Relative STANDARD ERRORS FOR FOUR ALTERnATIVE ASSET PRICING MODELS: US DATA, 10 SIZE-SORTED PORTFOLIOS.

The table presents the relative standard errors of $\pi$ GMM estimates under the Beta and the SDF methods, computed as $\sigma_{r}(\widehat{\pi})=\sigma(\widehat{\pi}) \div \mathrm{E}(\widehat{\pi})$. The returns and factors are generated under the null hypothesis with the factors sampled from the empirical distribution. Estimators decorated with * are from the Beta method; with A and B to the un-centred and centred SDF method; and with 1 and 2 to the first and second-stage respectively. The results are presented for different lengths of time series observations, and they are based on 10,000 simulations.

\begin{tabular}{|c|c|c|c|c|}
\hline$T$ & $\sigma_{r}\left(\widehat{\pi}_{1}^{A}\right) \div \sigma_{r}\left(\widehat{\pi}^{*}\right)$ & $\sigma_{r}\left(\widehat{\pi}_{2}^{A}\right) \div \sigma_{r}\left(\widehat{\pi}^{*}\right)$ & $\sigma_{r}\left(\widehat{\pi}_{1}^{B}\right) \div \sigma_{r}\left(\widehat{\pi}^{*}\right)$ & $\sigma_{r}\left(\widehat{\pi}_{2}^{B}\right) \div \sigma_{r}\left(\widehat{\pi}^{*}\right)$ \\
\hline & \multicolumn{4}{|c|}{ CAPM } \\
\hline 60 & 0.8247 & 1.1339 & 0.8234 & 1.0579 \\
\hline 360 & 0.8231 & 1.0056 & 0.8227 & 0.9992 \\
\hline 600 & 0.8215 & 0.9975 & 0.8214 & 0.9964 \\
\hline \multirow[t]{2}{*}{1000} & 0.8223 & 0.9941 & 0.8226 & 0.9947 \\
\hline & \multicolumn{4}{|c|}{ Fama-French } \\
\hline 60 & 0.6295 & 1.3624 & 0.6337 & 1.2610 \\
\hline 360 & 0.6967 & 1.0327 & 0.6978 & 0.9393 \\
\hline 600 & 0.7000 & 0.9485 & 0.7003 & 0.8968 \\
\hline \multirow[t]{2}{*}{1000} & 0.7478 & 0.9684 & 0.7479 & 0.9398 \\
\hline & \multicolumn{4}{|c|}{ RUH } \\
\hline 60 & 0.6414 & 1.2187 & 0.6429 & 1.1964 \\
\hline 360 & 0.6011 & 1.0345 & 0.6003 & 1.0045 \\
\hline 600 & 0.5991 & 1.0091 & 0.6003 & 0.9899 \\
\hline \multirow[t]{2}{*}{1000} & 0.6056 & 0.9871 & 0.6062 & 0.9789 \\
\hline & \multicolumn{4}{|c|}{ Carhart } \\
\hline 60 & 0.5773 & 1.2120 & 0.5785 & 1.1570 \\
\hline 360 & 0.6643 & 0.9810 & 0.6625 & 0.9169 \\
\hline 600 & 0.7399 & 0.9959 & 0.7396 & 0.9568 \\
\hline 1000 & 0.8548 & 1.0933 & 0.8557 & 1.0679 \\
\hline
\end{tabular}


Table 9: Specification tests for the CAPM model: US Data, 10 size-sorted PORTFOLIOS.

The table presents the expected value and the $\widehat{J}$ statistic under the Beta and the SDF methods. The specification tests are size tests. The first estimator decorated with * are from the Beta method; the second and third correspond to the first and second-stage un-centred SDF method; and the fourth and fifth to the first and second-stage centred SDF method. The results are presented for different lengths of time series observations, and they are based on 10,000 simulations.

\begin{tabular}{|c|c|c|c|c|c|c|c|c|c|c|c|c|c|c|c|}
\hline \multirow[b]{2}{*}{$T$} & \multicolumn{5}{|c|}{$1 \%$} & \multicolumn{5}{|c|}{$5 \%$} & \multicolumn{5}{|c|}{$10 \%$} \\
\hline & $\widehat{J}^{*}$ & $\widehat{J}_{1}^{A}$ & $\widehat{J}_{2}^{A}$ & $\widehat{J}_{1}^{B}$ & $\widehat{J}_{2}^{B}$ & $\widehat{J}^{*}$ & $\widehat{J}_{1}^{A}$ & $\widehat{J}_{2}^{A}$ & $\widehat{J}_{1}^{B}$ & $\widehat{J}_{2}^{B}$ & $\widehat{J}^{*}$ & $\widehat{J}_{1}^{A}$ & $\widehat{J}_{2}^{A}$ & $\widehat{J}_{1}^{B}$ & $\widehat{J}_{2}^{B}$ \\
\hline & \multicolumn{15}{|c|}{$W=S$} \\
\hline 60 & 3.39 & \multicolumn{2}{|c|}{5.42} & \multicolumn{2}{|c|}{6.13} & 9.57 & \multicolumn{2}{|c|}{15.07} & \multicolumn{2}{|c|}{15.99} & 15.72 & \multicolumn{2}{|c|}{23.20} & \multicolumn{2}{|c|}{24.31} \\
\hline 360 & 0.81 & \multicolumn{2}{|c|}{1.35} & \multicolumn{2}{|c|}{1.37} & 3.82 & \multicolumn{2}{|c|}{6.31} & \multicolumn{2}{|c|}{6.40} & 7.55 & \multicolumn{2}{|c|}{11.94} & \multicolumn{2}{|c|}{12.02} \\
\hline 600 & 0.60 & \multicolumn{2}{|c|}{1.10} & \multicolumn{2}{|c|}{1.14} & 3.44 & \multicolumn{2}{|c|}{5.65} & \multicolumn{2}{|c|}{5.68} & 6.91 & \multicolumn{2}{|c|}{10.72} & \multicolumn{2}{|c|}{10.79} \\
\hline 1000 & 0.58 & \multicolumn{2}{|c|}{1.03} & \multicolumn{2}{|c|}{1.02} & 3.12 & \multicolumn{2}{|c|}{5.26} & \multicolumn{2}{|c|}{5.30} & 6.59 & \multicolumn{2}{|c|}{10.37} & \multicolumn{2}{|c|}{10.34} \\
\hline & \multicolumn{15}{|c|}{$W=\mathrm{E}\left[r_{t} r_{t}^{\prime}\right]$} \\
\hline 60 & 0.12 & 0.29 & 0.67 & 0.55 & 1.24 & 1.68 & 3.26 & 4.68 & 4.75 & 6.35 & 4.39 & 8.11 & 9.86 & 10.69 & 12.70 \\
\hline 360 & 0.49 & 0.87 & 0.90 & 1.00 & 1.03 & 2.72 & 4.67 & 4.77 & 5.44 & 5.53 & 6.23 & 9.35 & 9.49 & 10.59 & 10.77 \\
\hline 600 & 0.43 & 0.79 & 0.84 & 1.00 & 1.02 & 2.92 & 4.75 & 4.79 & 5.25 & 5.29 & 6.00 & 9.28 & 9.39 & 10.20 & 10.28 \\
\hline \multirow[t]{2}{*}{1000} & 0.46 & 0.81 & 0.81 & 0.94 & 0.95 & 2.74 & 4.45 & 4.52 & 5.21 & 5.23 & 6.02 & 9.16 & 9.21 & 10.19 & 10.25 \\
\hline & \multicolumn{15}{|c|}{$W=\operatorname{Cov}\left[r_{t}\right]$} \\
\hline 60 & 3.39 & 4.91 & 5.22 & 6.69 & 7.26 & 9.57 & 13.88 & 14.29 & 16.92 & 17.68 & 15.72 & 21.24 & 22.02 & 25.55 & 26.28 \\
\hline 360 & 0.81 & 1.21 & 1.23 & 1.51 & 1.53 & 3.82 & 6.10 & 6.13 & 6.75 & 6.82 & 7.55 & 11.25 & 11.31 & 12.61 & 12.71 \\
\hline 600 & 0.60 & 1.05 & 1.06 & 1.28 & 1.32 & 3.44 & 5.39 & 5.40 & 6.06 & 6.08 & 6.91 & 10.13 & 10.16 & 11.36 & 11.40 \\
\hline 1000 & 0.58 & 0.95 & 0.96 & 1.12 & 1.13 & 3.12 & 4.98 & 4.99 & 5.65 & 5.66 & 6.59 & 9.76 & 9.78 & 10.87 & 10.87 \\
\hline
\end{tabular}



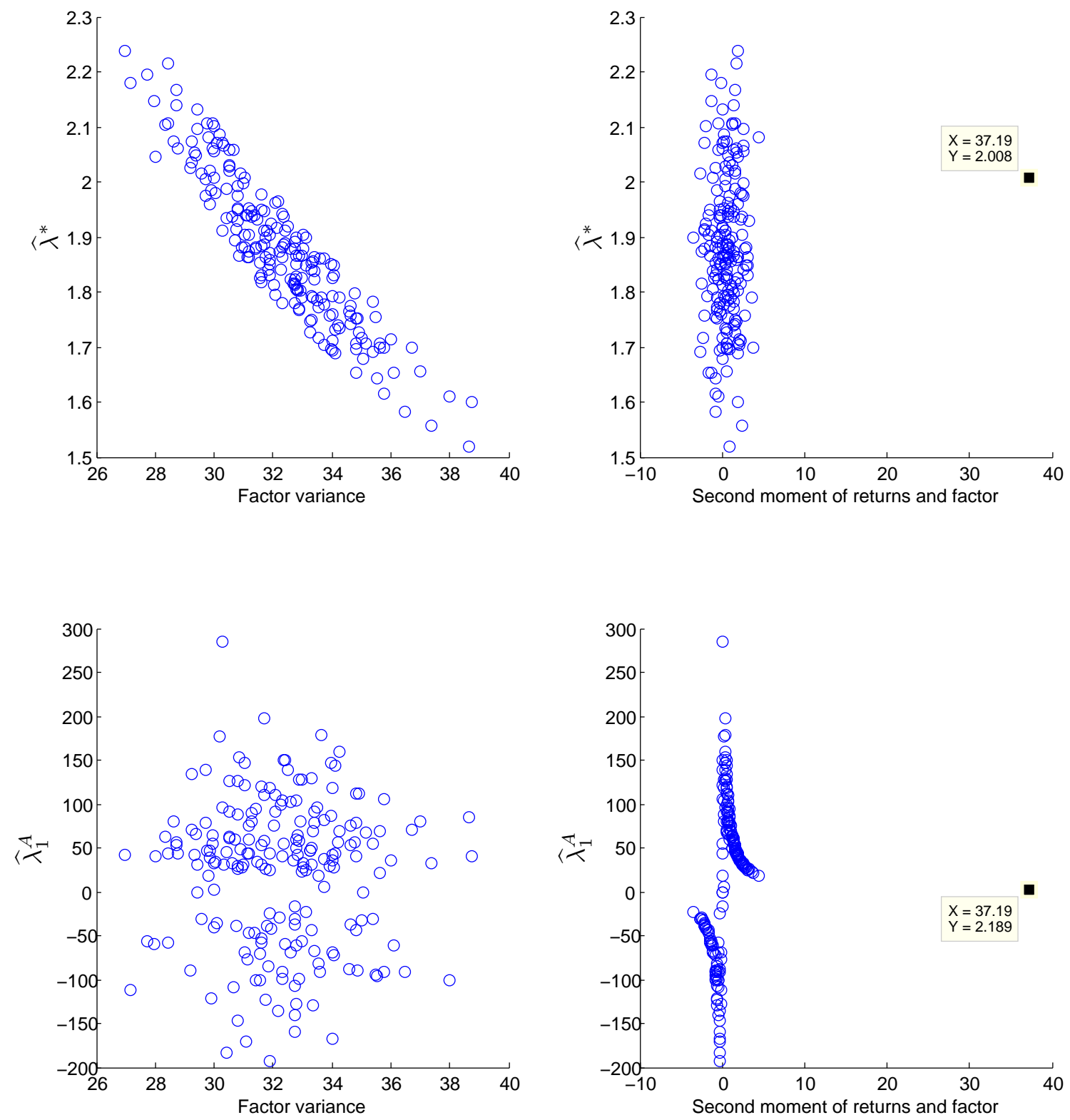

Figure 1: US: Estimators resulting from market factor draws with alternative variances and nearly independent of the portfolio returns. Actual values represented by the squared data cursor. 

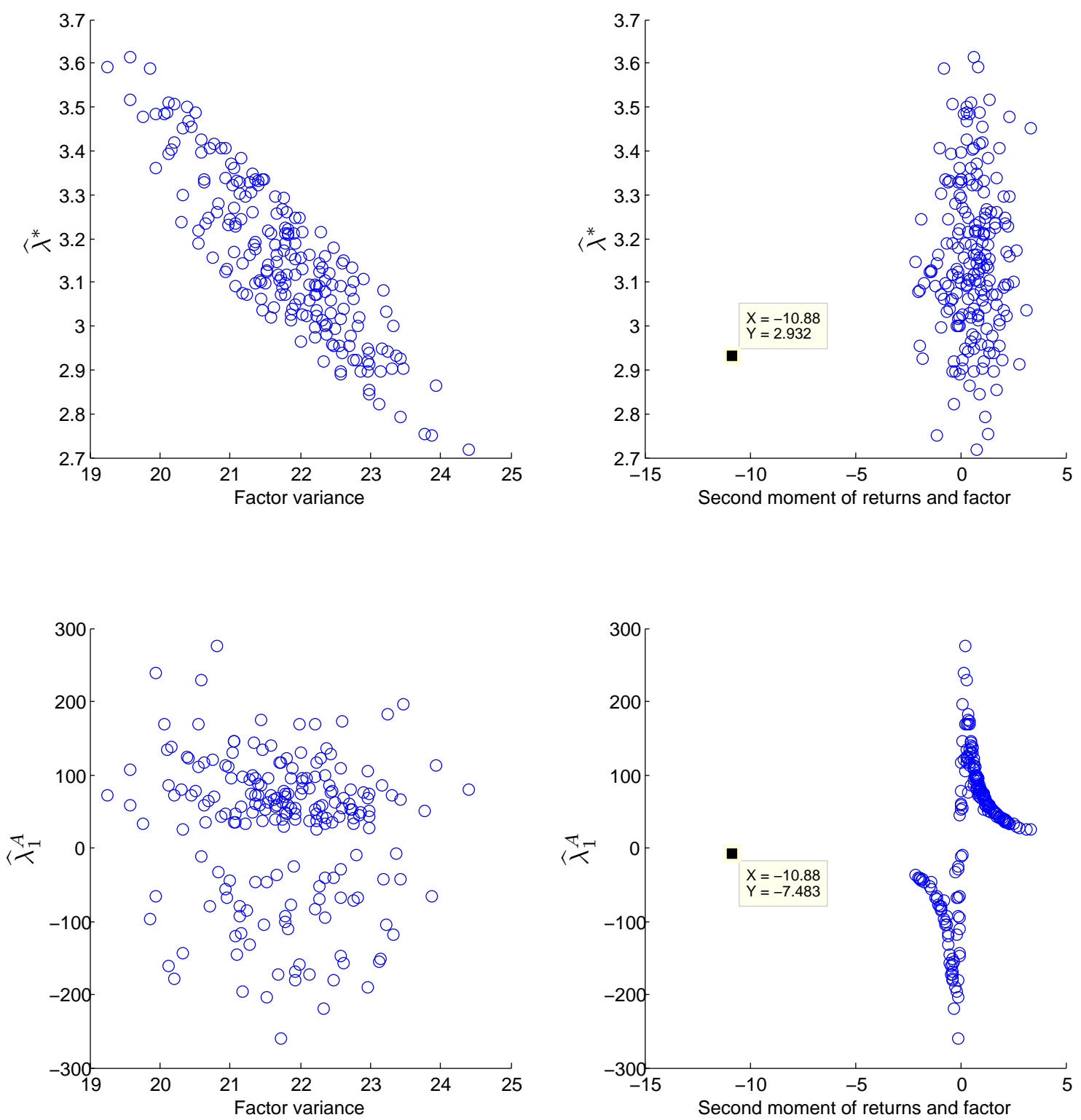

Figure 2: US: Estimators resulting from momentum factor draws with alternative variances and nearly independent of the portfolio returns. Actual values represented by the squared data cursor. 

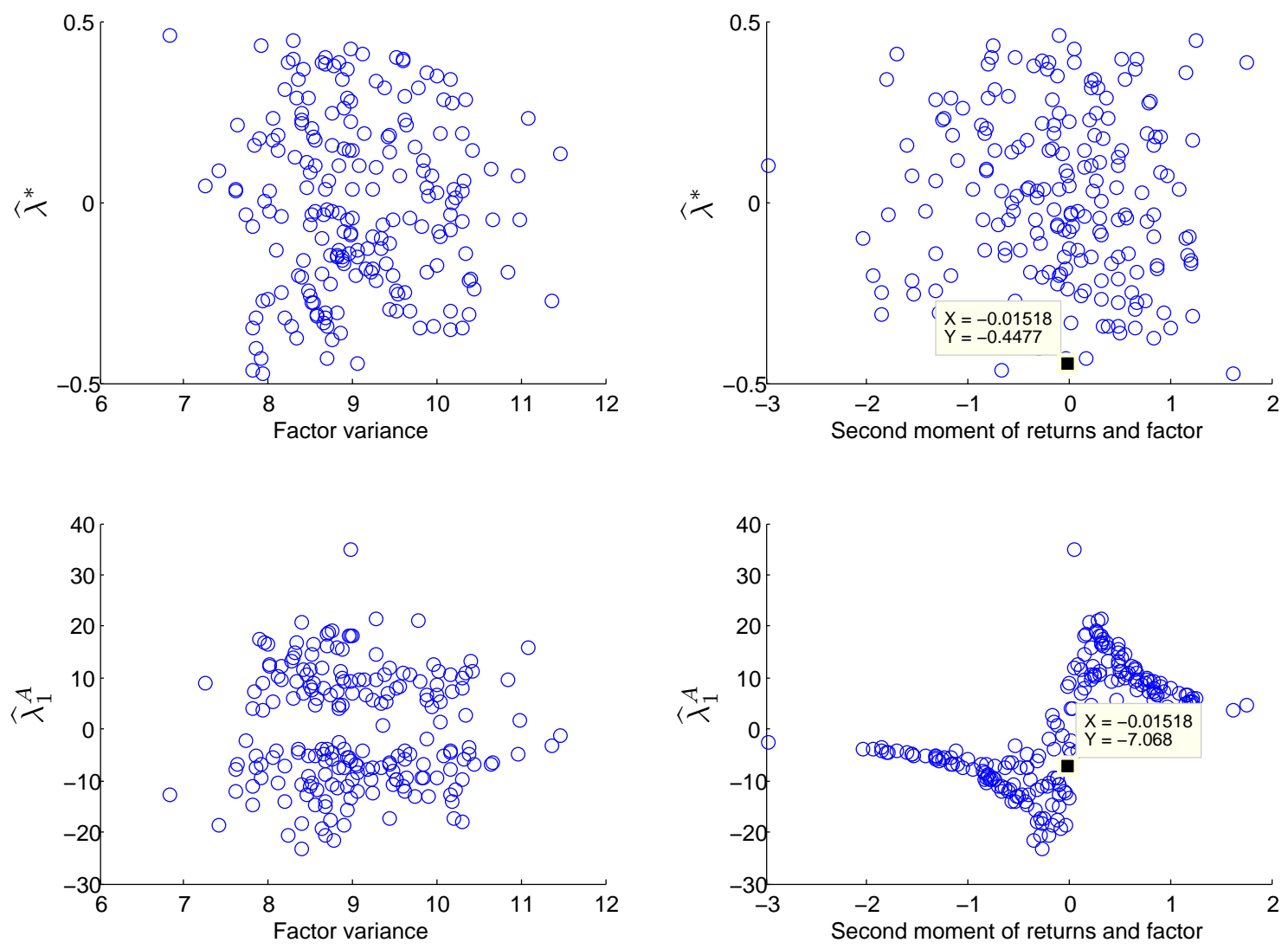

Figure 3: UK: Estimators resulting from size factor draws with alternative variances and nearly independent of the portfolio returns. Actual values represented by the squared data cursor. 
6 - Appendix

\section{Appendix}

Table 10: Descriptive statistics of Factors and portfolios.

US factors and portfolios taken from Kenneth R. French library, January 1927 to December $2009(T=996)$. UK factors and portfolios taken from Alan Gregory library, October 1980 to December 2008 ( $T=339)$.

\begin{tabular}{lccccccc}
\hline & \multicolumn{5}{c}{ Factors } & \multicolumn{3}{c}{ Portfolios } \\
\cline { 2 - 8 } & Market & Size & Value & Momentum & 10 size & 25 size-value & 30 industry \\
\hline Mean & 0.6098 & 0.2437 & 0.4036 & 0.6996 & 0.8547 & 0.8208 & 0.7088 \\
Variance & 29.9 & 11.18 & 12.93 & 23.37 & 0.0279 & 0.1080 & 0.0151 \\
Skewness & 0.17 & 2.17 & 1.83 & -3.03 & 0.0429 & -1.2789 & 0.2506 \\
Kurtosis & 10.51 & 24.99 & 18.45 & 29.43 & 2.9096 & 5.9788 & 2.5618 \\
\hline \multirow{2}{*}{ Mean } & 0.4262 & -0.04 & 0.5 & 0.8624 & 0.6237 & - & - \\
Variance & 21.44 & 9.06 & 10.82 & 14.95 & 0.1236 & - & - \\
Skewness & -1.1 & -0.19 & -0.14 & -0.5 & 1.6464 & - & - \\
Kurtosis & 6.93 & 3.93 & 7.79 & 6.79 & 4.3894 & - & - \\
\hline \hline
\end{tabular}


Table 11: Expected VAlue And Standard ERrors For FAMA-French MOdel: US DATA, 10 SIZE-SORTED PORTFOLIOS.

The table presents the expected value and the standard error of $\lambda$ GMM estimates under the Beta and the SDF methods. The returns and factors are generated under the null hypothesis with the factors sampled from the empirical distribution. The first estimator decorated with * are from the Beta method; the second and third correspond to the first and second-stage un-centred SDF method; and the fourth and fifth to the first and second-stage centred SDF method. The results are presented for different lengths of time series observations, and they are based on 10,000 simulations.

\begin{tabular}{lcccccccccc}
\hline \hline$T$ & $\mathrm{E}\left[\widehat{\lambda}^{*}\right]$ & $\mathrm{E}\left[\widehat{\lambda}_{1}^{A}\right]$ & $\mathrm{E}\left[\widehat{\lambda}_{2}^{A}\right]$ & $\mathrm{E}\left[\widehat{\lambda}_{1}^{B}\right]$ & $\mathrm{E}\left[\widehat{\lambda}_{2}^{B}\right]$ & $\sigma\left(\widehat{\lambda}^{*}\right)$ & $\sigma\left(\widehat{\lambda}_{1}^{A}\right)$ & $\sigma\left(\widehat{\lambda}_{2}^{A}\right)$ & $\sigma\left(\widehat{\lambda}_{1}^{B}\right)$ & $\sigma\left(\widehat{\lambda}_{2}^{B}\right)$ \\
\hline 60 & 2.00 & 2.17 & 2.63 & 2.44 & 2.68 & 2.69 & 2.84 & 3.37 & 3.24 & 3.54 \\
360 & 1.86 & 1.94 & 2.01 & 2.02 & 2.06 & 0.99 & 1.04 & 1.07 & 1.10 & 1.11 \\
600 & 1.83 & 1.90 & 1.94 & 1.96 & 1.99 & 0.75 & 0.79 & 0.80 & 0.83 & 0.83 \\
1000 & 1.82 & 1.89 & 1.92 & 1.95 & 1.97 & 0.57 & 0.61 & 0.60 & 0.64 & 0.63 \\
\hline & & & & \multicolumn{7}{c}{ Size } \\
\hline 60 & 1.06 & 1.87 & 2.16 & 2.38 & 2.56 & 4.13 & 5.84 & 6.28 & 6.25 & 6.41 \\
360 & 1.56 & 1.94 & 2.00 & 2.06 & 2.10 & 1.53 & 2.21 & 2.03 & 2.28 & 2.07 \\
600 & 1.60 & 1.93 & 1.96 & 2.02 & 2.05 & 1.17 & 1.68 & 1.52 & 1.72 & 1.55 \\
1000 & 1.66 & 1.95 & 1.97 & 2.03 & 2.05 & 0.90 & 1.29 & 1.17 & 1.33 & 1.20 \\
\hline & & & & \multicolumn{7}{c}{ Value } \\
\hline 60 & 2.40 & 3.03 & 3.47 & 2.67 & 2.45 & 3.77 & 10.92 & 9.70 & 11.62 & 10.05 \\
360 & 2.72 & 2.99 & 3.06 & 3.03 & 2.95 & 1.35 & 4.72 & 3.53 & 4.93 & 3.65 \\
600 & 2.74 & 2.95 & 3.00 & 3.02 & 2.97 & 1.05 & 3.61 & 2.64 & 3.76 & 2.74 \\
1000 & 2.76 & 2.98 & 3.01 & 3.05 & 3.02 & 0.79 & 2.78 & 2.05 & 2.88 & 2.13 \\
\hline \hline
\end{tabular}


$6-$ Appendix

Table 12: Expected VAlue AND STANDARD ERrors FOR RUH MOdel: US DATA, 10 SIZE-SORTED PORTFOLIOS.

The table presents the expected value and the standard error of $\lambda$ GMM estimates under the Beta and the SDF methods. The returns and factors are generated under the null hypothesis with the factors sampled from the empirical distribution. The first estimator decorated with * are from the Beta method; the second and third correspond to the first and second-stage un-centred SDF method; and the fourth and fifth to the first and second-stage centred SDF method. The results are presented for different lengths of time series observations, and they are based on 10,000 simulations.

\begin{tabular}{lcccccccccc}
\hline \hline$T$ & $\mathrm{E}\left[\widehat{\lambda}^{*}\right]$ & $\mathrm{E}\left[\widehat{\lambda}_{1}^{A}\right]$ & $\mathrm{E}\left[\widehat{\lambda}_{2}^{A}\right]$ & $\mathrm{E}\left[\widehat{\lambda}_{1}^{B}\right]$ & $\mathrm{E}\left[\widehat{\lambda}_{2}^{B}\right]$ & $\sigma\left(\widehat{\lambda}^{*}\right)$ & $\sigma\left(\widehat{\lambda}_{1}^{A}\right)$ & $\sigma\left(\widehat{\lambda}_{2}^{A}\right)$ & $\sigma\left(\widehat{\lambda}_{1}^{B}\right)$ & $\sigma\left(\widehat{\lambda}_{2}^{B}\right)$ \\
\hline 60 & 1.65 & 2.06 & 2.40 & 2.39 & 2.51 & 2.65 & 3.02 & 3.40 & 3.46 & 3.56 \\
360 & 1.70 & 1.87 & 1.94 & 1.99 & 2.02 & 0.96 & 1.04 & 1.06 & 1.11 & 1.11 \\
600 & 1.70 & 1.86 & 1.90 & 1.96 & 1.98 & 0.73 & 0.78 & 0.79 & 0.84 & 0.84 \\
1000 & 1.71 & 1.85 & 1.88 & 1.95 & 1.95 & 0.57 & 0.60 & 0.61 & 0.64 & 0.64 \\
\hline & & & & & Momentum & & & & \\
\hline 60 & 3.80 & 4.30 & 5.23 & 0.64 & 1.15 & 3.90 & 10.81 & 9.80 & 11.70 & 10.12 \\
360 & 2.75 & 2.90 & 3.12 & 2.12 & 2.34 & 1.57 & 4.78 & 4.33 & 5.03 & 4.45 \\
600 & 2.62 & 2.82 & 2.97 & 2.41 & 2.56 & 1.21 & 3.80 & 3.34 & 3.99 & 3.44 \\
1000 & 2.53 & 2.71 & 2.78 & 2.53 & 2.60 & 0.92 & 2.91 & 2.53 & 3.07 & 2.64 \\
\hline & & & & & & & & & \\
\hline 60 & 1.61 & 2.84 & 3.19 & 1.49 & 1.45 & 3.85 & 11.86 & 9.44 & 12.95 & 9.93 \\
360 & 2.40 & 2.93 & 3.00 & 2.65 & 2.71 & 1.36 & 4.45 & 3.43 & 4.73 & 3.63 \\
600 & 2.46 & 2.92 & 2.96 & 2.79 & 2.83 & 1.05 & 3.42 & 2.59 & 3.63 & 2.74 \\
1000 & 2.49 & 2.92 & 2.95 & 2.89 & 2.92 & 0.80 & 2.67 & 2.01 & 2.83 & 2.13 \\
\hline \hline
\end{tabular}


$6-$ Appendix

Table 13: Expected value and standard errors for Carhart model: US Data, 10 SIZE-SORTED PORTFOLIOS.

The table presents the expected value and the standard error of $\lambda$ GMM estimates under the Beta and the SDF methods. The returns and factors are generated under the null hypothesis with the factors sampled from the empirical distribution. The first estimator decorated with * are from the Beta method; the second and third correspond to the first and second-stage un-centred SDF method; and the fourth and fifth to the first and secondstage centred SDF method. The results are presented for different lengths of time series observations, and they are based on 10,000 simulations.

\begin{tabular}{|c|c|c|c|c|c|c|c|c|c|c|}
\hline$T$ & $\mathrm{E}\left[\widehat{\lambda}^{*}\right]$ & $\mathrm{E}\left[\widehat{\lambda}_{1}^{A}\right]$ & $\mathrm{E}\left[\widehat{\lambda}_{2}^{A}\right]$ & $\mathrm{E}\left[\widehat{\lambda}_{1}^{B}\right]$ & $\mathrm{E}\left[\widehat{\lambda}_{2}^{B}\right]$ & $\sigma\left(\widehat{\lambda}^{*}\right)$ & $\sigma\left(\widehat{\lambda}_{1}^{A}\right)$ & $\sigma\left(\widehat{\lambda}_{2}^{A}\right)$ & $\sigma\left(\widehat{\lambda}_{1}^{B}\right)$ & $\sigma\left(\widehat{\lambda}_{2}^{B}\right)$ \\
\hline & \multicolumn{10}{|c|}{ Market } \\
\hline 60 & 1.72 & 2.12 & 2.41 & 2.49 & 2.61 & 2.72 & 2.99 & 3.36 & 3.44 & 3.69 \\
\hline 360 & 1.67 & 1.88 & 1.93 & 2.01 & 2.03 & 0.98 & 1.05 & 1.07 & 1.14 & 1.14 \\
\hline 600 & 1.67 & 1.86 & 1.90 & 1.98 & 1.99 & 0.74 & 0.79 & 0.80 & 0.85 & 0.85 \\
\hline \multirow[t]{2}{*}{1000} & 1.66 & 1.85 & 1.87 & 1.95 & 1.95 & 0.57 & 0.61 & 0.61 & 0.65 & 0.65 \\
\hline & \multicolumn{10}{|c|}{ Size } \\
\hline 60 & -0.01 & 1.74 & 1.94 & 2.52 & 2.53 & 4.35 & 6.12 & 6.41 & 6.71 & 6.85 \\
\hline 360 & 1.05 & 1.88 & 1.92 & 2.16 & 2.12 & 1.57 & 2.21 & 2.08 & 2.31 & 2.17 \\
\hline 600 & 1.14 & 1.86 & 1.89 & 2.06 & 2.04 & 1.19 & 1.69 & 1.59 & 1.77 & 1.65 \\
\hline \multirow[t]{2}{*}{1000} & 1.21 & 1.90 & 1.92 & 2.06 & 2.04 & 0.90 & 1.28 & 1.19 & 1.34 & 1.24 \\
\hline & \multicolumn{10}{|c|}{ Value } \\
\hline 60 & 1.60 & 2.85 & 3.12 & 1.41 & 1.68 & 4.03 & 11.28 & 10.49 & 12.23 & 11.43 \\
\hline 360 & 2.29 & 2.89 & 2.96 & 2.49 & 2.72 & 1.41 & 4.53 & 3.92 & 4.81 & 4.17 \\
\hline 600 & 2.36 & 2.95 & 2.98 & 2.76 & 2.89 & 1.05 & 3.47 & 2.95 & 3.67 & 3.13 \\
\hline \multirow[t]{2}{*}{1000} & 2.38 & 2.88 & 2.90 & 2.81 & 2.90 & 0.80 & 2.69 & 2.29 & 2.85 & 2.43 \\
\hline & \multicolumn{10}{|c|}{ Momentum } \\
\hline 60 & 3.82 & 4.22 & 5.04 & 1.02 & 1.39 & 4.01 & 10.50 & 10.16 & 11.50 & 10.98 \\
\hline 360 & 2.68 & 2.95 & 3.12 & 2.39 & 2.48 & 1.59 & 4.67 & 4.38 & 4.97 & 4.58 \\
\hline 600 & 2.56 & 2.77 & 2.88 & 2.53 & 2.58 & 1.21 & 3.64 & 3.37 & 3.86 & 3.53 \\
\hline 1000 & 2.47 & 2.67 & 2.75 & 2.57 & 2.62 & 0.90 & 2.77 & 2.53 & 2.94 & 2.66 \\
\hline
\end{tabular}


$6-$ Appendix

Table 14: Expected value AND STANDARD ERrors FOR FOUR ALternative SingleFACTOR MODELS: US DATA, 10 SIZE-SORTED PORTFOLIOS.

The table presents the expected value and the standard error of $\lambda$ GMM estimates under the Beta and the SDF methods. The returns and factors are generated under the null hypothesis with the factors sampled from the empirical distribution. The first estimator decorated with * are from the Beta method; the second and third correspond to the first and second-stage un-centred SDF method; and the fourth and fifth to the first and secondstage centred SDF method. The results are presented for different lengths of time series observations, and they are based on 10,000 simulations.

\begin{tabular}{|c|c|c|c|c|c|c|c|c|c|c|}
\hline$T$ & $\mathrm{E}\left[\widehat{\lambda}^{*}\right]$ & $\mathrm{E}\left[\widehat{\lambda}_{1}^{A}\right]$ & $\mathrm{E}\left[\widehat{\lambda}_{2}^{A}\right]$ & $\mathrm{E}\left[\widehat{\lambda}_{1}^{B}\right]$ & $\mathrm{E}\left[\widehat{\lambda}_{2}^{B}\right]$ & $\sigma\left(\widehat{\lambda}^{*}\right)$ & $\sigma\left(\widehat{\lambda}_{1}^{A}\right)$ & $\sigma\left(\widehat{\lambda}_{2}^{A}\right)$ & $\sigma\left(\widehat{\lambda}_{1}^{B}\right)$ & $\sigma\left(\widehat{\lambda}_{2}^{B}\right)$ \\
\hline \multicolumn{11}{|c|}{ Single-factor model loaded with market factor } \\
\hline 60 & 2.14 & 2.18 & 2.89 & 2.34 & 2.72 & 2.62 & 2.86 & 3.55 & 3.15 & 3.49 \\
\hline 360 & 1.93 & 1.94 & 2.04 & 1.98 & 2.03 & 1.00 & 1.07 & 1.06 & 1.11 & 1.07 \\
\hline 600 & 1.93 & 1.93 & 1.99 & 1.96 & 1.99 & 0.77 & 0.83 & 0.80 & 0.86 & 0.82 \\
\hline 1000 & 1.92 & 1.92 & 1.95 & 1.95 & 1.96 & 0.59 & 0.64 & 0.61 & 0.66 & 0.62 \\
\hline \multicolumn{11}{|c|}{ Single-factor model loaded with size factor } \\
\hline 60 & 1.84 & 1.91 & 2.32 & 2.05 & 2.12 & 4.15 & 7.51 & 6.00 & 7.93 & 5.66 \\
\hline 360 & 1.98 & 2.03 & 2.07 & 2.06 & 2.04 & 1.57 & 2.62 & 1.76 & 2.67 & 1.75 \\
\hline 600 & 1.96 & 1.97 & 2.02 & 1.99 & 2.00 & 1.21 & 1.99 & 1.33 & 2.02 & 1.33 \\
\hline 1000 & 1.97 & 1.98 & 2.00 & 1.99 & 2.00 & 0.92 & 1.53 & 1.00 & 1.55 & 1.01 \\
\hline \multicolumn{11}{|c|}{ Single-factor model loaded with value factor } \\
\hline 60 & 3.01 & 3.06 & 3.72 & 3.57 & 2.33 & 3.80 & 14.54 & 8.59 & 15.52 & 8.17 \\
\hline 360 & 3.01 & 2.95 & 3.12 & 3.06 & 2.94 & 1.39 & 4.27 & 2.83 & 4.40 & 2.85 \\
\hline 600 & 3.00 & 3.01 & 3.09 & 3.09 & 3.00 & 1.06 & 3.23 & 2.17 & 3.32 & 2.21 \\
\hline 1000 & 3.01 & 2.98 & 3.05 & 3.03 & 3.01 & 0.81 & 2.46 & 1.62 & 2.52 & 1.65 \\
\hline \multicolumn{11}{|c|}{ Single-factor model loaded with momentum factor } \\
\hline 60 & 4.28 & 4.18 & 5.66 & 5.29 & 3.22 & 3.89 & 11.51 & 7.87 & 14.61 & 7.52 \\
\hline 360 & 3.00 & 2.99 & 3.27 & 3.15 & 2.98 & 1.70 & 3.06 & 2.77 & 3.31 & 2.75 \\
\hline 600 & 2.85 & 2.88 & 3.04 & 2.98 & 2.89 & 1.29 & 2.30 & 2.07 & 2.45 & 2.09 \\
\hline 1000 & 2.75 & 2.77 & 2.87 & 2.85 & 2.81 & 0.98 & 1.72 & 1.55 & 1.81 & 1.58 \\
\hline
\end{tabular}


$6-$ Appendix

Table 15: ExPECted VAlue AND STANDARD ERRORS FOR FOUR ALternative SingleASSET PRICING MODELS: US DATA, 10 SIZE-SORTED PORTFOLIOS.

The table presents the expected value and the standard error of $\pi$ GMM estimates under the Beta and the SDF methods. The returns and factors are generated under the null hypothesis with the factors sampled from the empirical distribution. The first estimator decorated with * are from the Beta method; the second and third correspond to the first and second-stage un-centred SDF method; and the fourth and fifth to the first and secondstage centred SDF method. The results are presented for different lengths of time series observations, and they are based on 10,000 simulations.

\begin{tabular}{|c|c|c|c|c|c|c|c|c|c|c|}
\hline$T$ & $\mathrm{E}\left[\widehat{\pi}^{*}\right]$ & $\mathrm{E}\left[\widehat{\pi}_{1}^{A}\right]$ & $\mathrm{E}\left[\widehat{\pi}_{2}^{A}\right]$ & $\mathrm{E}\left[\widehat{\pi}_{1}^{B}\right]$ & $\mathrm{E}\left[\widehat{\pi}_{2}^{B}\right]$ & $\sigma\left(\widehat{\pi}^{*}\right)$ & $\sigma\left(\widehat{\pi}_{1}^{A}\right)$ & $\sigma\left(\widehat{\pi}_{2}^{A}\right)$ & $\sigma\left(\widehat{\pi}_{1}^{B}\right)$ & $\sigma\left(\widehat{\pi}_{2}^{B}\right)$ \\
\hline \multicolumn{11}{|c|}{ Single-factor model loaded with market factor } \\
\hline 60 & 36.57 & 24.20 & 47.46 & 24.94 & 41.84 & 20.68 & 11.28 & 30.43 & 11.61 & 25.02 \\
\hline 360 & 15.14 & 10.03 & 15.56 & 10.17 & 15.45 & 8.63 & 4.70 & 8.92 & 4.77 & 8.80 \\
\hline 600 & 11.69 & 7.75 & 11.82 & 7.85 & 11.83 & 6.65 & 3.62 & 6.71 & 3.67 & 6.71 \\
\hline 1000 & 9.08 & 6.01 & 9.09 & 6.09 & 9.15 & 5.19 & 2.82 & 5.16 & 2.86 & 5.20 \\
\hline \multicolumn{11}{|c|}{ Single-factor model loaded with size factor } \\
\hline 60 & 64.25 & 29.57 & 59.35 & 30.23 & 59.01 & 41.46 & 16.19 & 38.59 & 16.73 & 38.45 \\
\hline 360 & 26.50 & 11.96 & 25.25 & 12.04 & 25.40 & 17.15 & 6.17 & 16.36 & 6.22 & 16.45 \\
\hline 600 & 20.33 & 9.15 & 19.45 & 9.21 & 19.55 & 13.07 & 4.69 & 12.53 & 4.72 & 12.59 \\
\hline 1000 & 15.78 & 7.12 & 15.11 & 7.16 & 15.19 & 10.30 & 3.70 & 9.87 & 3.72 & 9.91 \\
\hline \multicolumn{11}{|c|}{ Single-factor model loaded with value factor } \\
\hline 60 & 75.20 & 29.06 & 64.53 & 30.03 & 66.02 & 47.84 & 17.21 & 42.54 & 18.25 & 44.78 \\
\hline 360 & 30.89 & 11.11 & 25.40 & 11.27 & 25.73 & 19.75 & 5.64 & 16.08 & 5.74 & 16.32 \\
\hline 600 & 24.00 & 8.56 & 19.78 & 8.67 & 20.03 & 15.41 & 4.29 & 12.35 & 4.35 & 12.50 \\
\hline 1000 & 18.55 & 6.65 & 15.23 & 6.73 & 15.40 & 12.01 & 3.32 & 9.58 & 3.36 & 9.69 \\
\hline \multicolumn{11}{|c|}{ Single-factor model loaded with momentum factor } \\
\hline 60 & 74.60 & 25.48 & 61.76 & 27.17 & 64.01 & 47.87 & 13.21 & 40.38 & 15.04 & 43.14 \\
\hline 360 & 30.06 & 9.85 & 20.97 & 10.10 & 21.59 & 19.30 & 4.48 & 12.28 & 4.60 & 12.93 \\
\hline 600 & 23.60 & 7.64 & 15.98 & 7.81 & 16.47 & 15.04 & 3.44 & 9.11 & 3.52 & 9.45 \\
\hline 1000 & 18.10 & 5.91 & 12.29 & 6.03 & 12.60 & 11.56 & 2.67 & 6.98 & 2.72 & 7.19 \\
\hline
\end{tabular}


$6-$ Appendix

Table 16: Relative STANDARD ERRORS FOR FOUR ALTERNATIVE SINGLE-ASSET PRICING MODELS: US DATA, 10 SIZE-SORTED PORTFOLIOS.

The table presents the relative standard errors of $\pi$ GMM estimates under the Beta and the SDF methods, computed as $\sigma_{r}(\widehat{\pi})=\sigma(\widehat{\pi}) \div \mathrm{E}(\widehat{\pi})$. The returns and factors are generated under the null hypothesis with the factors sampled from the empirical distribution. Estimators decorated with * are from the Beta method; with A and B to the un-centred and centred SDF method; and with 1 and 2 to the first and second-stage respectively. The results are presented for different lengths of time series observations, and they are based on 10,000 simulations.

\begin{tabular}{|c|c|c|c|c|}
\hline$T$ & $\sigma_{r}\left(\widehat{\pi}_{1}^{A}\right) \div \sigma_{r}\left(\widehat{\pi}^{*}\right)$ & $\sigma_{r}\left(\widehat{\pi}_{2}^{A}\right) \div \sigma_{r}\left(\widehat{\pi}^{*}\right)$ & $\sigma_{r}\left(\widehat{\pi}_{1}^{B}\right) \div \sigma_{r}\left(\widehat{\pi}^{*}\right)$ & $\sigma_{r}\left(\widehat{\pi}_{2}^{B}\right) \div \sigma_{r}\left(\widehat{\pi}^{*}\right)$ \\
\hline & \multicolumn{4}{|c|}{ CAPM } \\
\hline 60 & 0.8247 & 1.1339 & 0.8234 & 1.0579 \\
\hline 360 & 0.8231 & 1.0056 & 0.8227 & 0.9992 \\
\hline 600 & 0.8215 & 0.9975 & 0.8214 & 0.9964 \\
\hline \multirow[t]{2}{*}{1000} & 0.8223 & 0.9941 & 0.8226 & 0.9947 \\
\hline & \multicolumn{4}{|c|}{ CAPM loaded with size factor } \\
\hline 60 & 0.8483 & 1.0076 & 0.8578 & 1.0099 \\
\hline 360 & 0.7975 & 1.0009 & 0.7979 & 1.0006 \\
\hline 600 & 0.7978 & 1.0023 & 0.7979 & 1.0015 \\
\hline \multirow[t]{2}{*}{1000} & 0.7969 & 1.0010 & 0.7970 & 1.0007 \\
\hline & \multicolumn{4}{|c|}{ CAPM loaded with value factor } \\
\hline 60 & 0.9311 & 1.0363 & 0.9554 & 1.0662 \\
\hline 360 & 0.7943 & 0.9902 & 0.7968 & 0.9924 \\
\hline 600 & 0.7800 & 0.9723 & 0.7803 & 0.9717 \\
\hline \multirow[t]{2}{*}{1000} & 0.7719 & 0.9724 & 0.7720 & 0.9719 \\
\hline & \multicolumn{4}{|c|}{ CAPM loaded with momentum factor } \\
\hline 60 & 0.8083 & 1.0190 & 0.8627 & 1.0503 \\
\hline 360 & 0.7082 & 0.9118 & 0.7098 & 0.9329 \\
\hline 600 & 0.7071 & 0.8945 & 0.7079 & 0.8999 \\
\hline 1000 & 0.7073 & 0.8892 & 0.7076 & 0.8929 \\
\hline
\end{tabular}


Table 17: Specification tests for the Fama-French model: US Data, 10 SizeSORTED PORTFOLIOS.

The table presents the expected value and the $\widehat{J}$ statistic under the Beta and the SDF methods. The specification tests are size tests. The first estimator decorated with ${ }^{*}$ are from the Beta method; the second and third correspond to the first and second-stage un-centred SDF method; and the fourth and fifth to the first and second-stage centred SDF method. The results are presented for different lengths of time series observations, and they are based on 10,000 simulations.

\begin{tabular}{|c|c|c|c|c|c|c|c|c|c|c|c|c|c|c|c|}
\hline \multirow[b]{2}{*}{$T$} & \multicolumn{5}{|c|}{$1 \%$} & \multicolumn{5}{|c|}{$5 \%$} & \multicolumn{5}{|c|}{$10 \%$} \\
\hline & $\widehat{J}^{*}$ & $\widehat{J}_{1}^{A}$ & $\widehat{J}_{2}^{A}$ & $\widehat{J}_{1}^{B}$ & $\widehat{J}_{2}^{B}$ & $\widehat{J}^{*}$ & $\widehat{J}_{1}^{A}$ & $\widehat{J}_{2}^{A}$ & $\widehat{J}_{1}^{B}$ & $\widehat{J}_{2}^{B}$ & $\widehat{J}^{*}$ & $\widehat{J}_{1}^{A}$ & $\widehat{J}_{2}^{A}$ & $\widehat{J}_{1}^{B}$ & $\widehat{J}_{2}^{B}$ \\
\hline & \multicolumn{15}{|c|}{$W=S$} \\
\hline 60 & 0.66 & \multicolumn{2}{|c|}{3.02} & \multicolumn{2}{|c|}{3.76} & 2.43 & \multicolumn{2}{|c|}{9.91} & \multicolumn{2}{|c|}{11.01} & 4.80 & \multicolumn{2}{|c|}{16.59} & \multicolumn{2}{|c|}{17.89} \\
\hline 360 & 0.16 & \multicolumn{2}{|c|}{1.17} & \multicolumn{2}{|c|}{1.22} & 1.10 & \multicolumn{2}{|c|}{5.62} & \multicolumn{2}{|c|}{5.75} & 2.63 & \multicolumn{2}{|c|}{10.56} & \multicolumn{2}{|c|}{10.61} \\
\hline 600 & 0.18 & \multicolumn{2}{|c|}{1.03} & \multicolumn{2}{|c|}{1.03} & 1.06 & \multicolumn{2}{|c|}{5.20} & \multicolumn{2}{|c|}{5.22} & 2.53 & \multicolumn{2}{|c|}{10.16} & \multicolumn{2}{|c|}{10.25} \\
\hline 1000 & 0.11 & \multicolumn{2}{|c|}{1.07} & \multicolumn{2}{|c|}{1.08} & 0.93 & \multicolumn{2}{|c|}{5.10} & \multicolumn{2}{|c|}{5.25} & 2.34 & \multicolumn{2}{|c|}{10.07} & \multicolumn{2}{|c|}{10.10} \\
\hline & \multicolumn{15}{|c|}{$W=\mathrm{E}\left[r_{t} r_{t}^{\prime}\right]$} \\
\hline 60 & 0.02 & 0.39 & 0.98 & 1.11 & 2.47 & 0.28 & 3.31 & 5.12 & 6.82 & 10.27 & 0.93 & 7.61 & 10.76 & 14.17 & 18.51 \\
\hline 360 & 0.10 & 0.79 & 0.84 & 1.36 & 1.41 & 0.82 & 4.56 & 4.84 & 6.21 & 6.50 & 2.03 & 9.16 & 9.42 & 11.93 & 12.19 \\
\hline 600 & 0.13 & 0.87 & 0.91 & 1.30 & 1.30 & 0.85 & 4.50 & 4.60 & 5.86 & 6.01 & 2.18 & 8.98 & 9.05 & 11.60 & 11.78 \\
\hline \multirow[t]{2}{*}{1000} & 0.10 & 0.76 & 0.78 & 1.26 & 1.29 & 0.80 & 4.44 & 4.49 & 5.81 & 5.86 & 2.14 & 9.14 & 9.20 & 11.37 & 11.42 \\
\hline & \multicolumn{15}{|c|}{$W=\operatorname{Cov}\left[r_{t}\right]$} \\
\hline 60 & 0.66 & 3.25 & 3.78 & 6.70 & 7.97 & 2.43 & 9.74 & 11.25 & 17.28 & 19.16 & 4.80 & 16.46 & 18.48 & 25.30 & 27.42 \\
\hline 360 & 0.16 & 1.14 & 1.21 & 1.84 & 1.89 & 1.10 & 5.61 & 5.67 & 7.32 & 7.47 & 2.63 & 10.50 & 10.63 & 13.39 & 13.62 \\
\hline 600 & 0.18 & 1.03 & 1.04 & 1.68 & 1.71 & 1.06 & 5.08 & 5.12 & 6.56 & 6.59 & 2.53 & 9.64 & 9.70 & 12.53 & 12.65 \\
\hline 1000 & 0.11 & 0.92 & 0.94 & 1.46 & 1.46 & 0.93 & 4.76 & 4.78 & 6.20 & 6.22 & 2.34 & 9.58 & 9.62 & 11.88 & 11.90 \\
\hline
\end{tabular}


Table 18: Specification tests for the RUH model: US Data, 10 size-sorted PORTFOLIOS.

The table presents the expected value and the $\widehat{J}$ statistic under the Beta and the SDF methods. The specification tests are size tests. The first estimator decorated with * are from the Beta method; the second and third correspond to the first and second-stage un-centred SDF method; and the fourth and fifth to the first and second-stage centred SDF method. The results are presented for different lengths of time series observations, and they are based on 10,000 simulations.

\begin{tabular}{|c|c|c|c|c|c|c|c|c|c|c|c|c|c|c|c|}
\hline \multirow[b]{2}{*}{$T$} & \multicolumn{5}{|c|}{$1 \%$} & \multicolumn{5}{|c|}{$5 \%$} & \multicolumn{5}{|c|}{$10 \%$} \\
\hline & $\widehat{J}^{*}$ & $\widehat{J}_{1}^{A}$ & $\widehat{J}_{2}^{A}$ & $\widehat{J}_{1}^{B}$ & $\widehat{J}_{2}^{B}$ & $\widehat{J}^{*}$ & $\widehat{J}_{1}^{A}$ & $\widehat{J}_{2}^{A}$ & $\widehat{J}_{1}^{B}$ & $\widehat{J}_{2}^{B}$ & $\widehat{J}^{*}$ & $\widehat{J}_{1}^{A}$ & $\widehat{J}_{2}^{A}$ & $\widehat{J}_{1}^{B}$ & $\widehat{J}_{2}^{B}$ \\
\hline & \multicolumn{15}{|c|}{$W=S$} \\
\hline 60 & 0.58 & \multicolumn{2}{|c|}{1.92} & \multicolumn{2}{|c|}{2.62} & 2.31 & \multicolumn{2}{|c|}{7.04} & \multicolumn{2}{|c|}{8.35} & 4.44 & \multicolumn{2}{|c|}{12.50} & \multicolumn{2}{|c|}{14.24} \\
\hline 360 & 0.14 & \multicolumn{2}{|c|}{0.81} & \multicolumn{2}{|c|}{0.90} & 0.88 & \multicolumn{2}{|c|}{4.40} & \multicolumn{2}{|c|}{4.77} & 2.16 & \multicolumn{2}{|c|}{8.71} & \multicolumn{2}{|c|}{9.14} \\
\hline 600 & 0.13 & \multicolumn{2}{|c|}{0.84} & \multicolumn{2}{|c|}{0.98} & 0.88 & \multicolumn{2}{|c|}{4.68} & \multicolumn{2}{|c|}{5.02} & 2.35 & \multicolumn{2}{|c|}{9.37} & \multicolumn{2}{|c|}{9.82} \\
\hline 1000 & 0.12 & \multicolumn{2}{|c|}{0.83} & \multicolumn{2}{|c|}{0.87} & 0.79 & \multicolumn{2}{|c|}{4.36} & \multicolumn{2}{|c|}{4.55} & 1.97 & \multicolumn{2}{|c|}{8.93} & \multicolumn{2}{|c|}{9.13} \\
\hline & \multicolumn{15}{|c|}{$W=\mathrm{E}\left[r_{t} r_{t}^{\prime}\right]$} \\
\hline 60 & 0.03 & 0.30 & 1.16 & 1.05 & 2.83 & 0.21 & 2.88 & 5.17 & 6.41 & 9.98 & 0.80 & 6.62 & 10.24 & 13.39 & 18.05 \\
\hline 360 & 0.06 & 0.55 & 0.65 & 1.29 & 1.39 & 0.63 & 3.83 & 4.10 & 5.85 & 6.08 & 1.68 & 8.01 & 8.36 & 11.20 & 11.72 \\
\hline 600 & 0.09 & 0.74 & 0.79 & 1.39 & 1.42 & 0.72 & 4.15 & 4.32 & 6.69 & 6.83 & 1.94 & 8.65 & 8.91 & 11.94 & 12.16 \\
\hline \multirow[t]{2}{*}{1000} & 0.12 & 0.61 & 0.62 & 1.11 & 1.13 & 0.70 & 3.78 & 3.91 & 6.00 & 6.10 & 1.75 & 8.04 & 8.15 & 11.37 & 11.49 \\
\hline & \multicolumn{15}{|c|}{$W=\operatorname{Cov}\left[r_{t}\right]$} \\
\hline 60 & 0.58 & 2.81 & 3.90 & 6.28 & 7.81 & 2.31 & 8.68 & 10.94 & 16.48 & 19.08 & 4.44 & 15.21 & 17.64 & 24.71 & 27.88 \\
\hline 360 & 0.14 & 0.85 & 0.99 & 1.82 & 1.92 & 0.88 & 4.66 & 4.88 & 6.98 & 7.23 & 2.16 & 9.04 & 9.36 & 12.80 & 13.14 \\
\hline 600 & 0.13 & 0.89 & 0.95 & 1.59 & 1.69 & 0.88 & 4.71 & 4.90 & 7.26 & 7.32 & 2.35 & 9.27 & 9.50 & 12.93 & 13.13 \\
\hline 1000 & 0.12 & 0.69 & 0.71 & 1.30 & 1.31 & 0.79 & 4.05 & 4.11 & 6.29 & 6.37 & 1.97 & 8.51 & 8.60 & 11.84 & 11.92 \\
\hline
\end{tabular}


Table 19: Specification tests for the Carhart model: US data, 10 size-sorted PORTFOLIOS.

The table presents the expected value and the $\widehat{J}$ statistic under the Beta and the SDF methods. The specification tests are size tests. The first estimator decorated with * are from the Beta method; the second and third correspond to the first and second-stage un-centred SDF method; and the fourth and fifth to the first and second-stage centred SDF method. The results are presented for different lengths of time series observations, and they are based on 10,000 simulations.

\begin{tabular}{|c|c|c|c|c|c|c|c|c|c|c|c|c|c|c|c|}
\hline \multirow[b]{2}{*}{$T$} & \multicolumn{5}{|c|}{$1 \%$} & \multicolumn{5}{|c|}{$5 \%$} & \multicolumn{5}{|c|}{$10 \%$} \\
\hline & $\widehat{J}^{*}$ & $\widehat{J}_{1}^{A}$ & $\widehat{J}_{2}^{A}$ & $\widehat{J}_{1}^{B}$ & $\widehat{J}_{2}^{B}$ & $\widehat{J}^{*}$ & $\widehat{J}_{1}^{A}$ & $\widehat{J}_{2}^{A}$ & $\widehat{J}_{1}^{B}$ & $\widehat{J}_{2}^{B}$ & $\widehat{J}^{*}$ & $\widehat{J}_{1}^{A}$ & $\widehat{J}_{2}^{A}$ & $\widehat{J}_{1}^{B}$ & $\widehat{J}_{2}^{B}$ \\
\hline & \multicolumn{15}{|c|}{$W=S$} \\
\hline 60 & 0.19 & 1.77 & 1.77 & 2.60 & 2.60 & 0.87 & 6.88 & 6.88 & 8.41 & 8.41 & 2.09 & 12.71 & 12.71 & 14.19 & 14.19 \\
\hline 360 & 0.04 & 0.97 & 0.97 & 1.11 & 1.11 & 0.45 & 5.02 & 5.02 & 5.31 & 5.31 & 1.36 & 9.74 & 9.74 & 9.87 & 9.87 \\
\hline 600 & 0.10 & 0.89 & 0.89 & 0.98 & 0.98 & 0.54 & 4.42 & 4.42 & 4.63 & 4.63 & 1.24 & 9.26 & 9.26 & 9.56 & 9.56 \\
\hline \multirow[t]{2}{*}{1000} & 0.07 & 0.88 & 0.88 & 0.96 & 0.96 & 0.44 & 4.79 & 4.79 & 4.96 & 4.96 & 1.15 & 9.83 & 9.83 & 10.03 & 10.03 \\
\hline & \multicolumn{15}{|c|}{$W=\mathrm{E}\left[r_{t} r_{t}^{\prime}\right]$} \\
\hline 60 & 0.00 & 0.28 & 1.20 & 1.44 & 3.55 & 0.04 & 3.11 & 5.80 & 7.97 & 12.40 & 0.26 & 7.70 & 11.45 & 15.57 & 20.83 \\
\hline 360 & 0.02 & 0.73 & 0.85 & 1.62 & 1.76 & 0.28 & 4.33 & 4.66 & 7.10 & 7.45 & 0.97 & 8.76 & 9.27 & 12.41 & 12.89 \\
\hline 600 & 0.07 & 0.72 & 0.80 & 1.43 & 1.49 & 0.42 & 3.82 & 3.97 & 6.11 & 6.34 & 1.08 & 8.17 & 8.38 & 11.95 & 12.26 \\
\hline \multirow[t]{2}{*}{1000} & 0.04 & 0.75 & 0.77 & 1.46 & 1.50 & 0.38 & 4.25 & 4.32 & 6.31 & 6.47 & 1.02 & 8.64 & 8.80 & 12.17 & 12.41 \\
\hline & \multicolumn{15}{|c|}{$W=\operatorname{Cov}\left[r_{t}\right]$} \\
\hline 60 & 0.19 & 2.29 & 3.40 & 6.57 & 8.45 & 0.87 & 8.58 & 10.50 & 16.81 & 19.76 & 2.09 & 14.26 & 17.03 & 25.16 & 28.72 \\
\hline 360 & 0.04 & 1.06 & 1.14 & 2.07 & 2.27 & 0.45 & 5.02 & 5.32 & 8.15 & 8.45 & 1.36 & 9.79 & 10.21 & 13.72 & 14.13 \\
\hline 600 & 0.10 & 0.91 & 0.95 & 1.71 & 1.76 & 0.54 & 4.29 & 4.41 & 6.78 & 7.07 & 1.24 & 8.70 & 8.92 & 12.73 & 12.94 \\
\hline 1000 & 0.07 & 0.78 & 0.82 & 1.61 & 1.68 & 0.44 & 4.48 & 4.54 & 6.71 & 6.78 & 1.15 & 9.03 & 9.21 & 12.64 & 12.77 \\
\hline
\end{tabular}



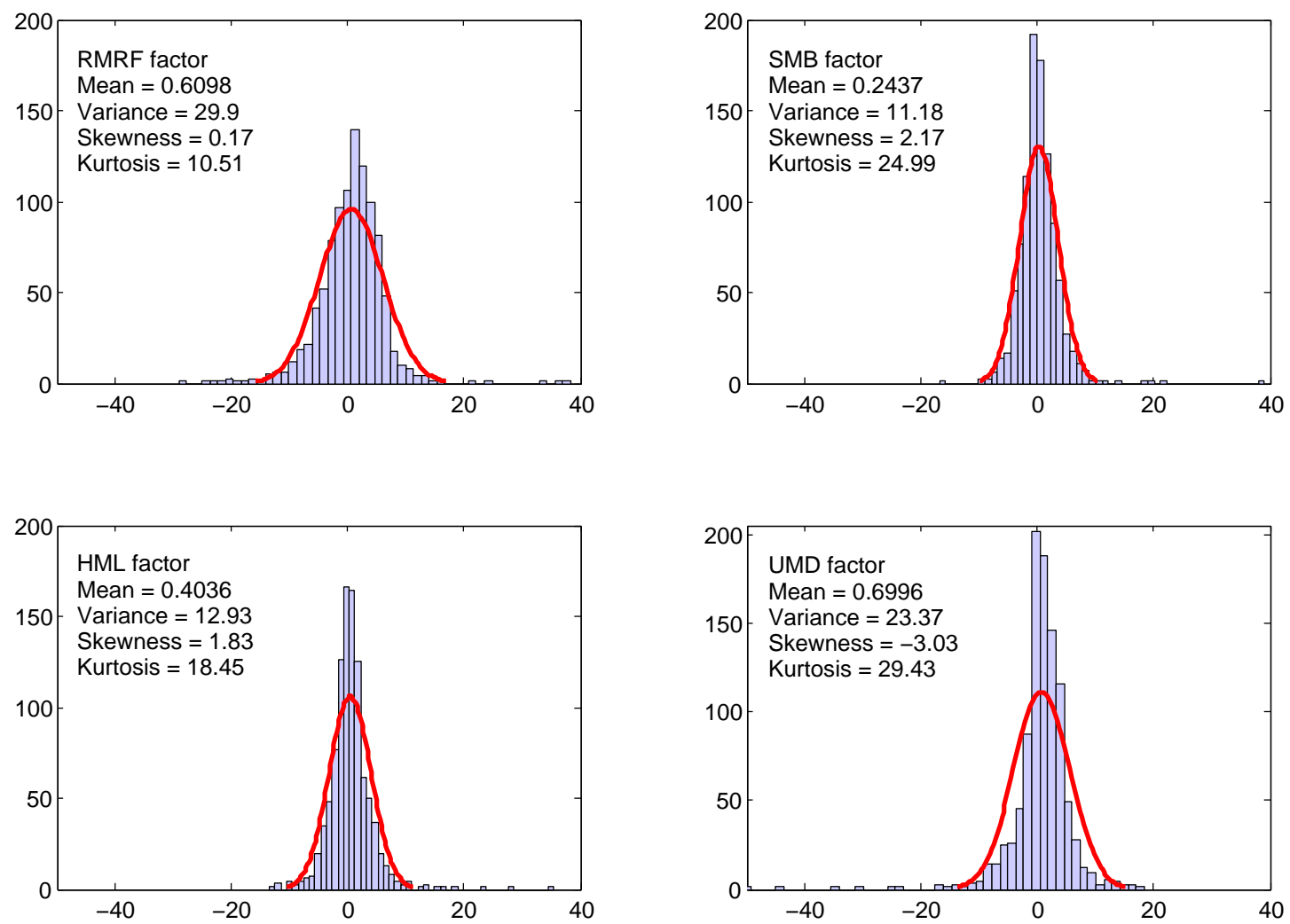

Figure 4: Histograms for the US factors with a fitted normal distribution, $T=996$ from January 1927 to December 2009. Data from Kenneth R. French library. 

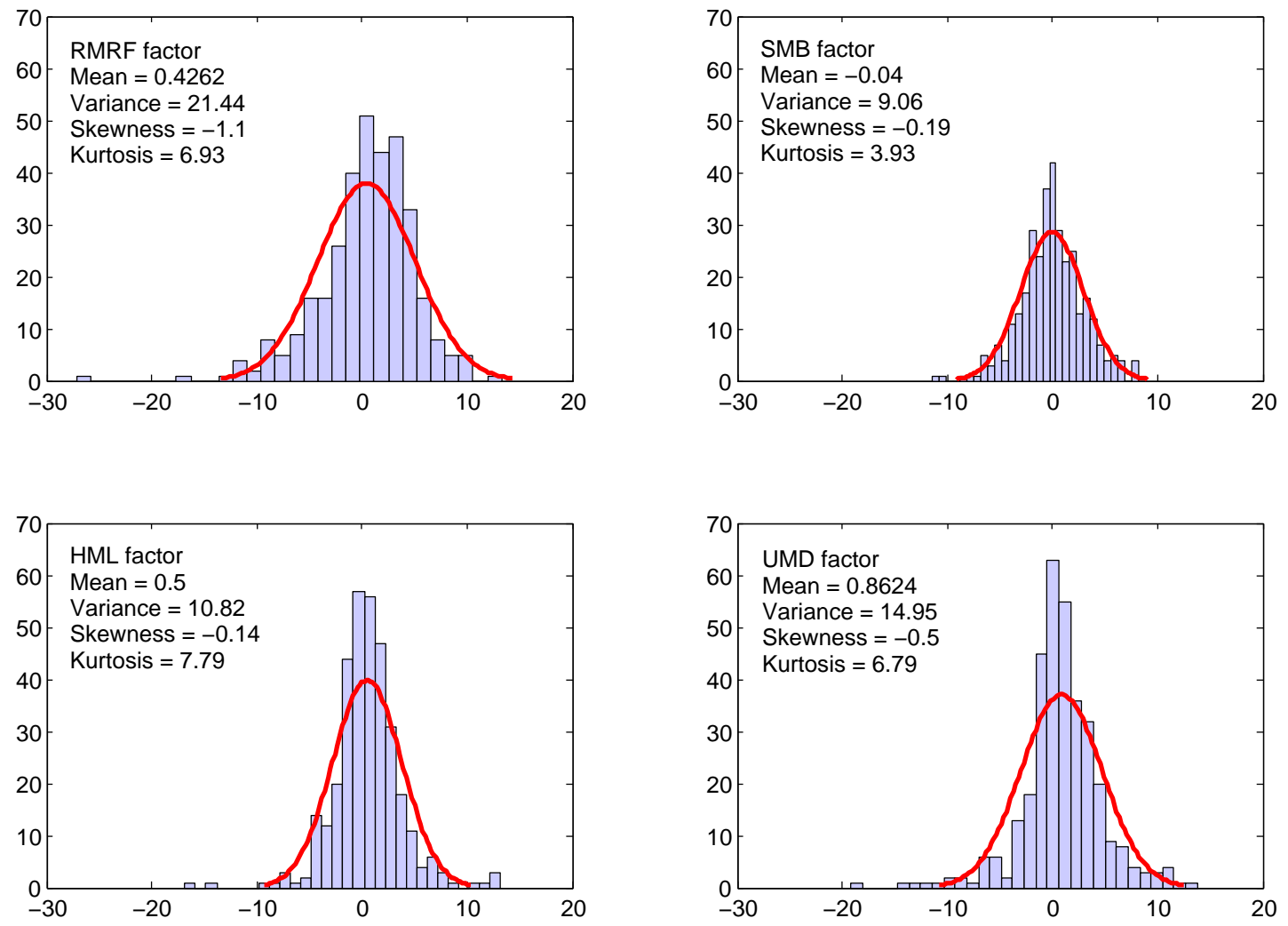

Figure 5: Histograms for the UK factors with a fitted normal distribution, $T=339$ from October 1980 to December 2008. Data from Alan Gregory library. 

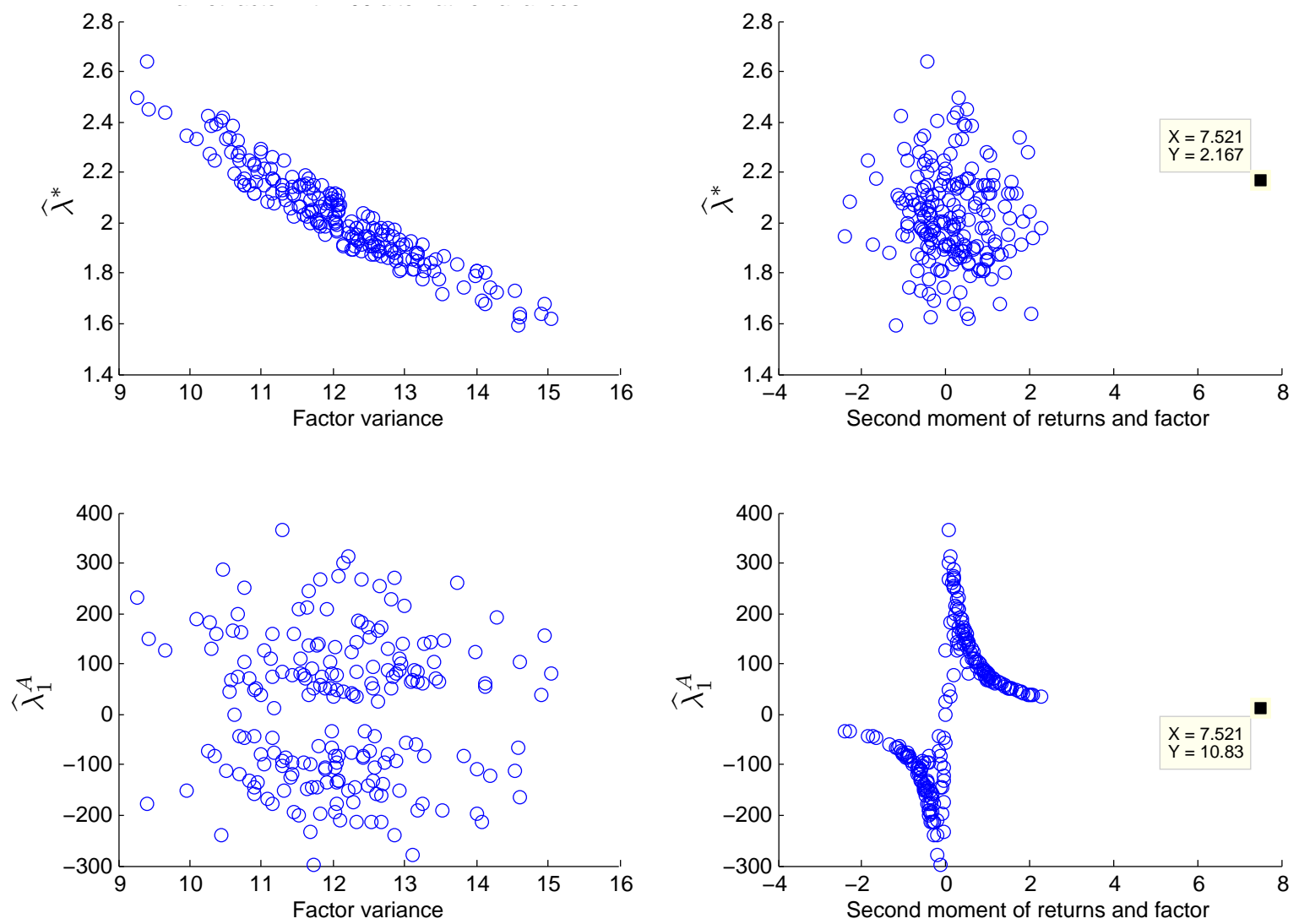

Figure 6: US: Estimators resulting from size factor draws with alternative variances and nearly independent of the portfolio returns. Actual values represented by the squared data cursor. 

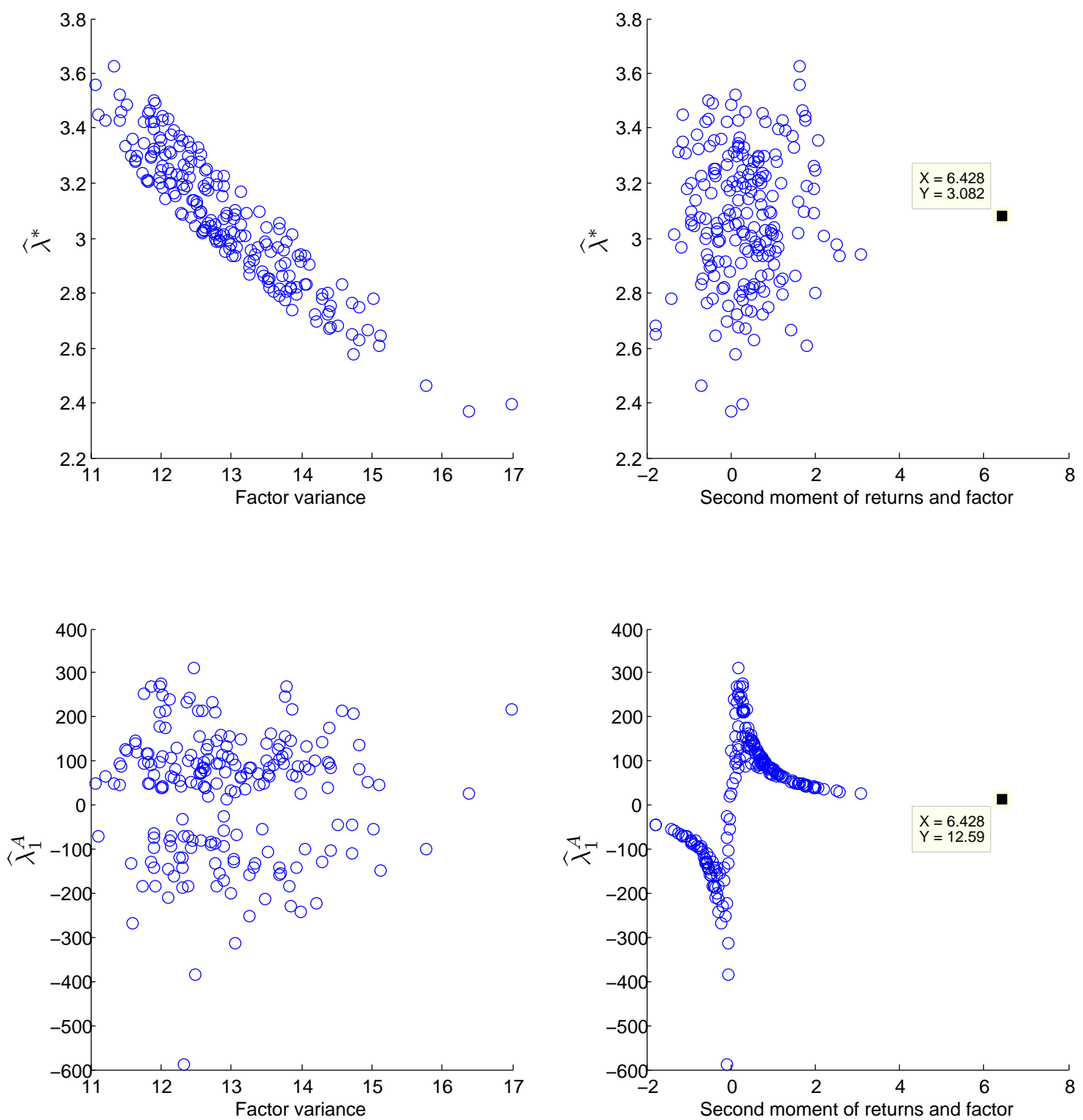

Figure 7: US: Estimators resulting from value factor draws with alternative variances and nearly independent of the portfolio returns. Actual values represented by the squared data cursor. 

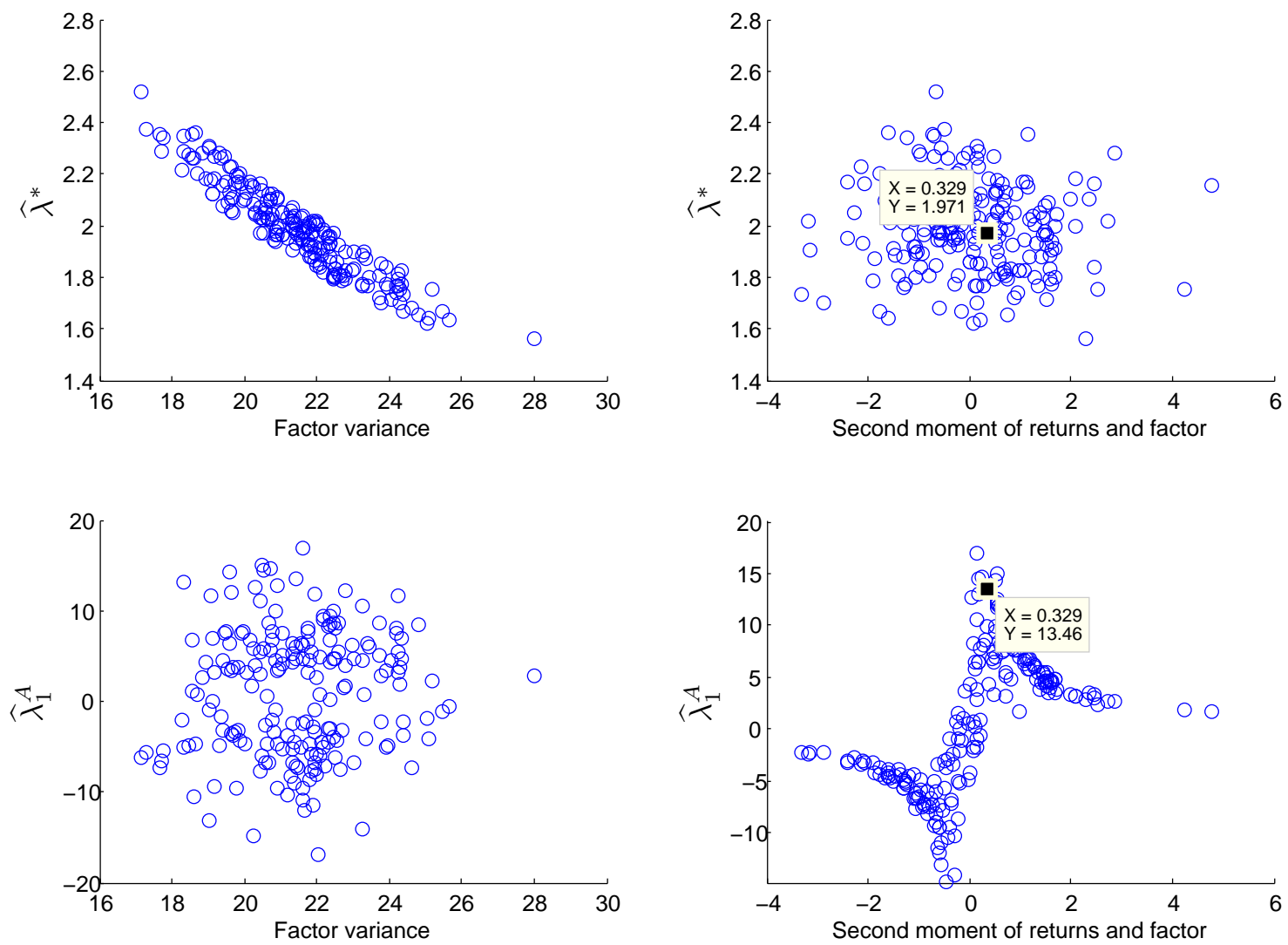

Figure 8: UK: Estimators resulting from market factor draws with alternative variances and nearly independent of the portfolio returns. Actual values represented by the squared data cursor. 

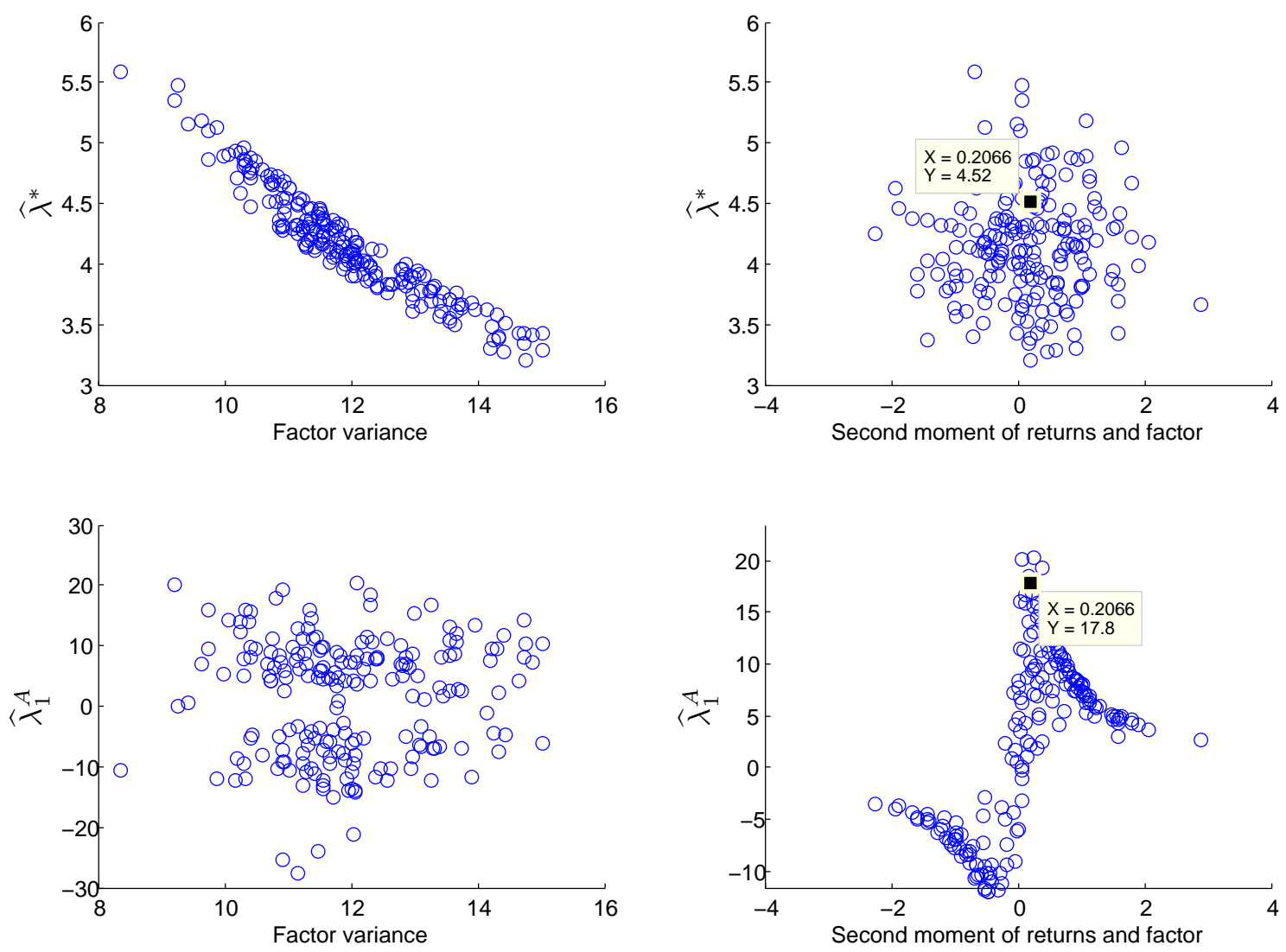

Figure 9: UK: Estimators resulting from value factor draws with alternative variances and nearly independent of the portfolio returns. Actual values represented by the squared data cursor. 

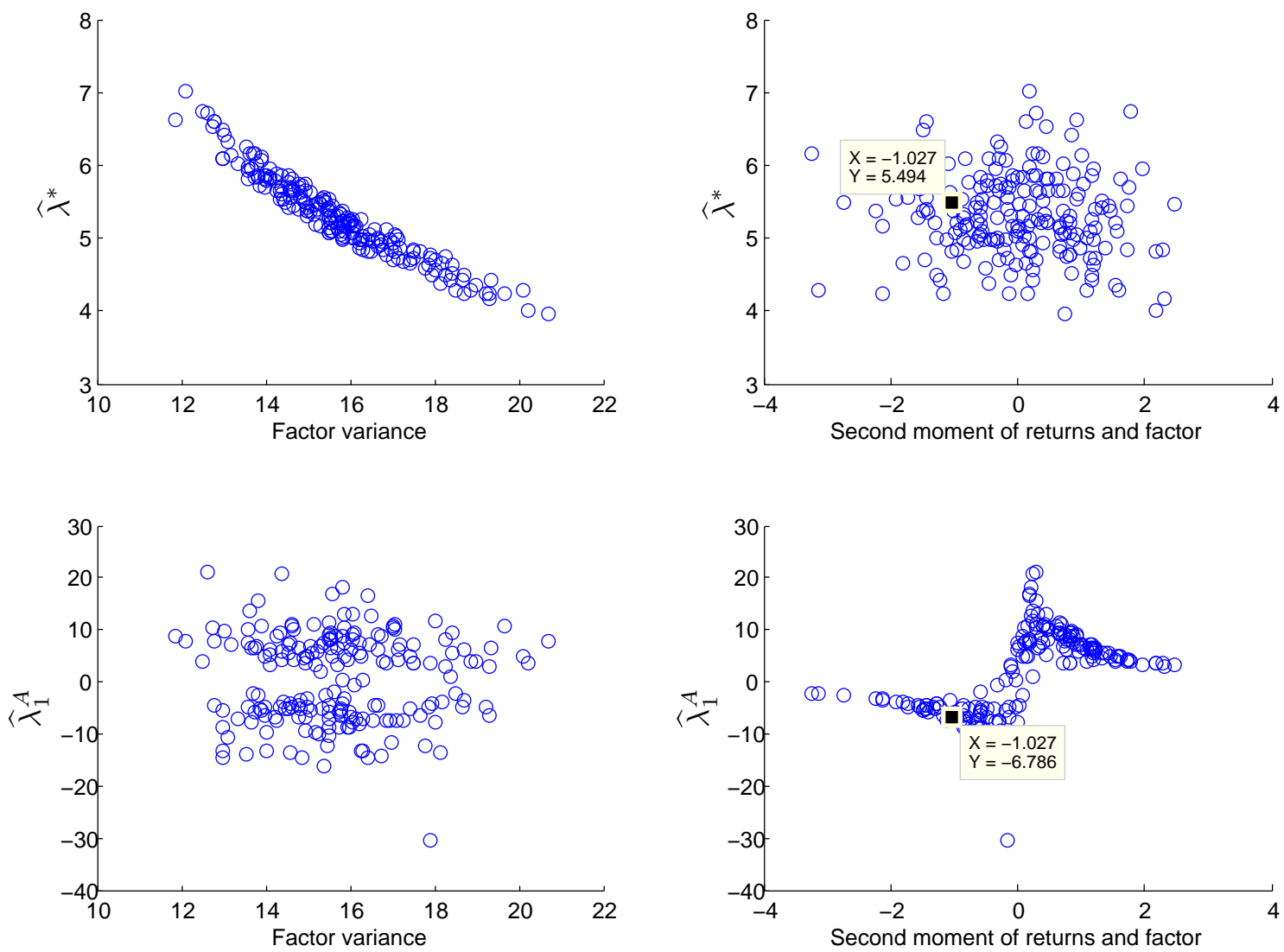

Figure 10: UK: Estimators resulting from momentum factor draws with alternative variances and nearly independent of the portfolio returns. Actual values represented by the squared data cursor. 